\title{
How to Draw Tropical Planes
}

\author{
Sven Herrmann* \\ Department of Mathematics \\ Technische Universität Darmstadt, Germany \\ sherrmann@mathematik.tu-darmstadt.de \\ Courant Research Center \\ Anders Jensen ${ }^{\dagger}$ \\ Georg-August-Universität Göttingen, Germany \\ jensen@uni-math.gwdg.de \\ Michael Joswig $\ddagger$ \\ Department of Mathematics \\ Technische Universität Darmstadt, Germany \\ joswig@mathematik.tu-darmstadt .de \\ Bernd Sturmfels ${ }^{\S}$ \\ Department of Mathematics \\ University of California, Berkeley, USA \\ bernd@math . berkeley. edu \\ Submitted: Sep 1, 2008; Accepted: Apr 14, 2009; Published: Apr 20, 2009 \\ Mathematics Subject Classification: 52B40 (14M15, 05C05) \\ Dedicated to Anders Björner on the occasion of his 60th birthday.
}

\begin{abstract}
The tropical Grassmannian parameterizes tropicalizations of ordinary linear spaces, while the Dressian parameterizes all tropical linear spaces in $\mathbb{T P}^{n-1}$. We study these parameter spaces and we compute them explicitly for $n \leq 7$. Planes are identified with matroid subdivisions and with arrangements of trees. These representations are then used to draw pictures.
\end{abstract}

\section{Introduction}

A line in tropical projective space $\mathbb{T} \mathbb{P}^{n-1}$ is an embedded metric tree which is balanced and has $n$ unbounded edges pointing into the coordinate directions. The parameter space of these objects is the tropical Grassmannian $\operatorname{Gr}(2, n)$. This is a simplicial fan [29], known to evolutionary biologists as the space of phylogenetic trees with $n$ labeled leaves $[24, \S 3.5]$, and known to algebraic geometers as the moduli space of rational tropical curves [23].

\footnotetext{
*This author was supported by a Graduate Grant of TU Darmstadt.

$\dagger$ This author was supported by a Sofia Kovalevskaja prize awarded to Olga Holtz at TU Berlin.

†This author was supported by the DFG Research Unit "Polyhedral Surfaces".

$\S$ This author was supported by an Alexander-von-Humboldt senior award at TU Berlin and the US National Science Foundation.
} 
Speyer [27, 28] introduced higher-dimensional tropical linear spaces. They are contractible polyhedral complexes all of whose maximal cells have the same dimension $d-1$. Among these are the realizable tropical linear spaces which arise from $(d-1)$-planes in classical projective space $\mathbb{P}_{\mathbb{K}}^{n-1}$ over a field $\mathbb{K}$ with a non-archimedean valuation. Realizable linear spaces are parameterized by the tropical Grassmannian $\operatorname{Gr}(d, n)$, as shown in [29]. Note that, as a consequence of [29, Theorem 3.4] and [27, Proposition 2.2], all tropical lines $(d=2)$ are realizable. Tropical Grassmannians represent compact moduli spaces of hyperplane arrangements. Introduced by Alexeev, Hacking, Keel, and Tevelev $[1,16,21]$, these objects are natural generalizations of the moduli space $\bar{M}_{0, n}$.

In this paper we focus on the case $d=3$. By a tropical plane we mean a twodimensional tropical linear subspace of $\mathbb{T P}^{n-1}$. It was shown in $[29, \S 5]$ that all tropical planes are realizable when $n \leq 6$. This result rests on the classification of planes in $\mathbb{T} \mathbb{P}^{5}$ which is shown in Figure 1. We here derive the analogous complete picture of what is possible for $n=7$. In Theorem 3.6, we show that for larger $n$ most tropical planes are not realizable. More precisely, the dimension of $\operatorname{Dr}(3, n)$ grows quadratically with $n$, while the dimension of $\operatorname{Gr}(3, n)$ is only linear in $n$.

Tropical linear spaces are represented by vectors of Plücker coordinates. The axioms characterizing such vectors were discovered two decades ago by Andreas Dress who called them valuated matroids. We therefore propose the name Dressian for the tropical prevariety $\operatorname{Dr}(d, n)$ which parameterizes $(d-1)$-dimensional tropical linear spaces in $\mathbb{T} \mathbb{P}^{n-1}$. The purpose of this paper is to gather results about $\operatorname{Dr}(3, n)$ which may be used in the future to derive general structural information about all Dressians and Grassmannians.

The paper is organized as follows. In Section 2 we review the formal definition of the Dressian and the Grassmannian, and we present our results on $\operatorname{Gr}(3,7)$ and $\operatorname{Dr}(3,7)$. These also demonstrate the remarkable scope of current software for tropical geometry. In particular, we use Gfan [18] for computing tropical varieties and polymake [13] for computations in polyhedral geometry.

Tropical planes are dual to regular matroid subdivisions of the hypersimplex $\Delta(3, n)$. The theory of these subdivisions is developed in Section 3, after a review of matroid basics, and this allows us to prove various combinatorial results about the Dressian $\operatorname{Dr}(3, n)$. With a specific construction of matroid subdivisions of the hypersimplices which arise from the set of lines in finite projective spaces over $\mathbb{G F}(2)$ these combinatorial results yield the lower bound on the dimensions of the Dressians in Theorem 3.6.

A main contribution is the bijection between tropical planes and arrangements of metric trees in Theorem 4.4. This bijection tropicalizes the following classical picture. Every plane $\mathbb{P}_{\mathbb{K}}^{n-1}$ corresponds to an arrangement of $n$ lines in $\mathbb{P}_{\mathbb{K}}^{2}$, and hence to a rank3 -matroid on $n$ elements. Lines are now replaced by trees, and arrangements of trees are used to encode matroid subdivisions. These can be non-regular, as shown in Section 4. A key step in the proof of Theorem 4.4 is Proposition 4.3 which compares the two natural fan structures on $\operatorname{Dr}(3, n)$, one arising from the structure as a tropical prevariety, the other from the secondary fan of the hypersimplex $\Delta(3, n)$. It turns out that they coincide. The Section 5 answers the question in the title of this paper, and, in particular, it explains the seven diagrams in Figure 1 and their 94 analogs for $n=7$. In Section 6 we extend 

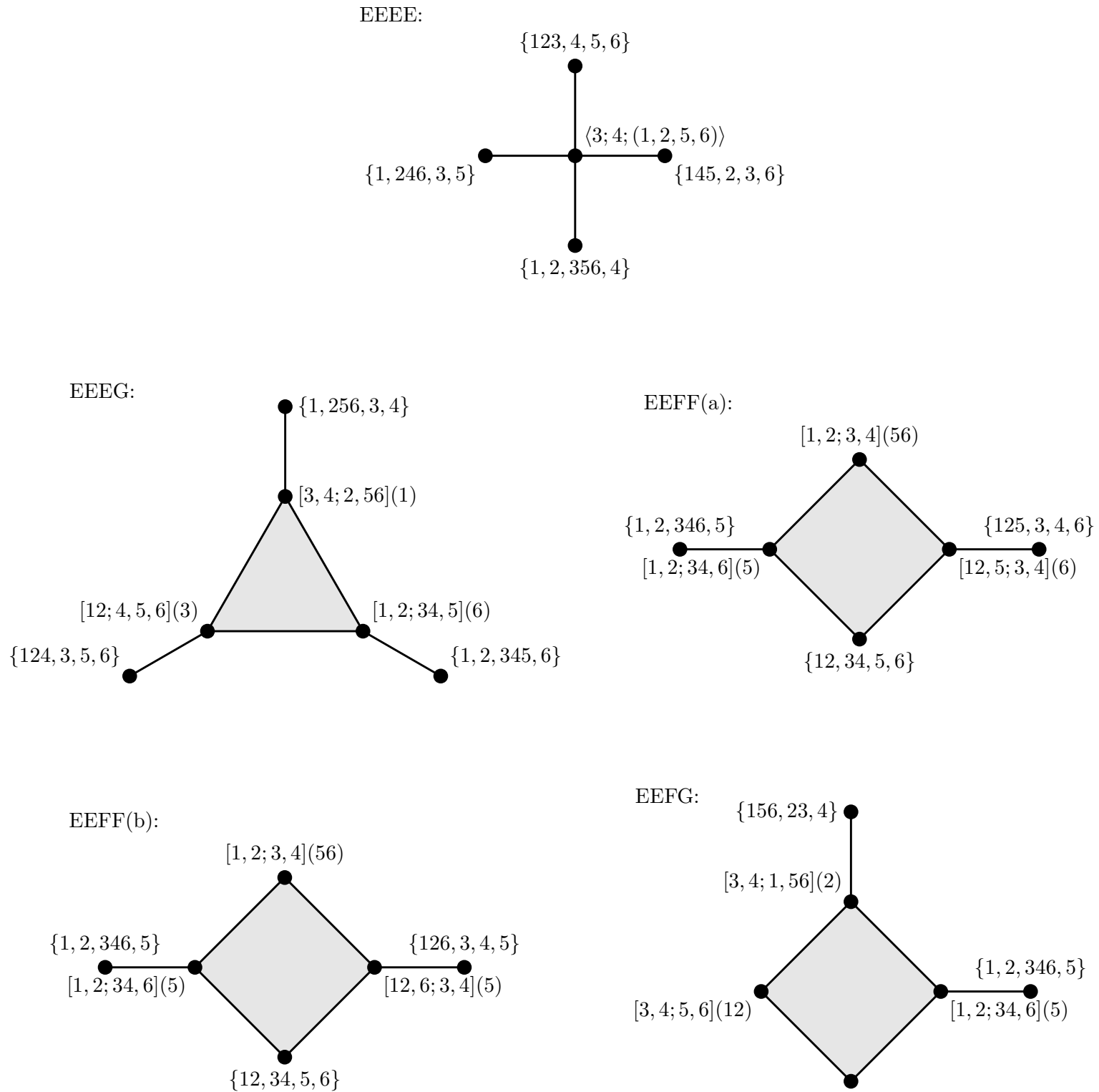

EEFG:

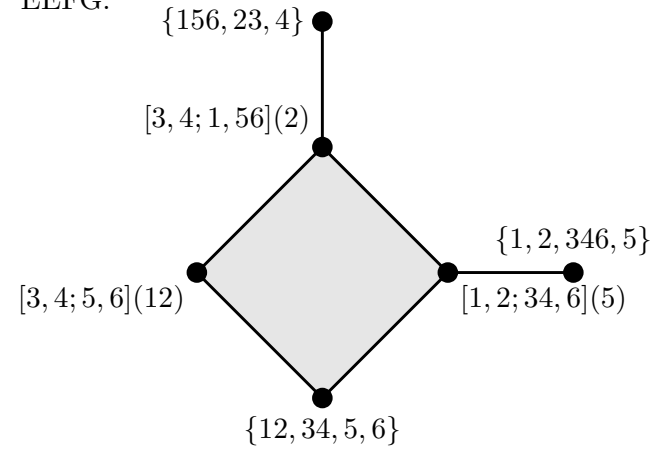

EFFG:

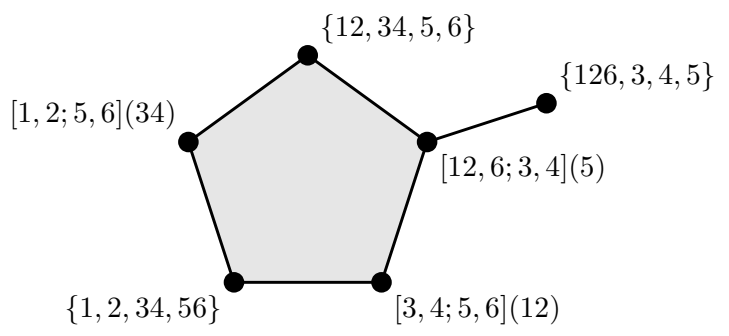

FFFGG:

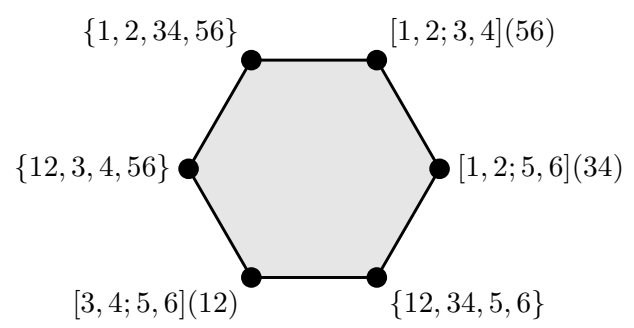

Figure 1: The seven types of generic tropical planes in $\mathbb{T P}^{5}$. 
the notion of Grassmannians and Dressians from $\Delta(d, n)$ to arbitrary matroid polytopes.

We are indebted to Francisco Santos, David Speyer, Walter Wenzel, Lauren Williams, and an anonymous referee for their helpful comments.

\section{Computations}

Let $I$ be a homogeneous ideal in the polynomial ring $\mathbb{K}\left[x_{1}, \ldots, x_{t}\right]$ over a field $\mathbb{K}$. Each vector $\lambda \in \mathbb{R}^{t}$ gives rise to a partial term order and thus defines an initial ideal $\operatorname{in}_{\lambda}(I)$, by choosing terms of lowest weight for each polynomial in $I$. The set of all initial ideals of $I$ induces a fan structure on $\mathbb{R}^{t}$. This is the Gröbner fan of $I$, which can be computed using Gfan [18]. The subfan induced by those initial ideals which do not contain any monomial is the tropical variety $\mathbb{T}(I)$. If $I$ is a principal ideal then $\mathbb{T}(I)$ is a tropical hypersurface. A tropical prevariety is the intersection of finitely many tropical hypersurfaces. Each tropical variety is a tropical prevariety, but the converse does not hold [25, Lemma 3.7].

Consider a fixed $d \times n$-matrix of indeterminates. Then each $d \times d$-minor is defined by selecting $d$ columns $\left\{i_{1}, i_{2}, \ldots, i_{d}\right\}$. Denoting the corresponding minor $p_{i_{1} \ldots i_{d}}$, the algebraic relations among all $d \times d$-minors define the Plücker ideal $I_{d, n}$ in $\mathbb{K}\left[p_{S}\right]$, where $S$ ranges over $\left(\begin{array}{c}{[n]} \\ d\end{array}\right)$, the set of all $d$-element subsets of $[n]:=\{1,2, \ldots, n\}$. The ideal $I_{d, n}$ is a homogeneous prime ideal. The tropical Grassmannian $\operatorname{Gr}(d, n)$ is the tropical variety of the Plücker ideal $I_{d, n}$. Among the generators of $I_{d, n}$ are the three term Plücker relations

$$
p_{S i j} p_{S k l}-p_{S i k} p_{S j l}+p_{S i l} p_{S j k}
$$

where $S \in\left(\begin{array}{c}{[n]} \\ d-2\end{array}\right)$ and $i, j, k, l \in[n] \backslash S$ pairwise distinct. Here $S i j$ is shorthand notation for the set $S \cup\{i, j\}$. The relations (1) do not generate the Plücker ideal $I_{d, n}$ for $d \geq 3$, but they always suffice to generate the image of $I_{d, n}$ in the Laurent polynomial ring $\mathbb{K}\left[p_{S}^{ \pm 1}\right]$.

The Dressian $\operatorname{Dr}(d, n)$ is the tropical prevariety defined by all three term Plücker relations. The elements of $\operatorname{Dr}(d, n)$ are the finite tropical Plücker vectors of Speyer [27]. A general tropical Plücker vector is allowed to have $\infty$ as a coordinate, while a finite one is not. The three term relations define a natural Plücker fan structure on the Dressian $\operatorname{Dr}(d, n)$ : two weight vectors $\lambda$ and $\lambda^{\prime}$ are in the same cone if they specify the same initial form for each trinomial (1). In Sections 3 and 4 we shall derive an alternative description of the Dressian $\operatorname{Dr}(d, n)$ and its Plücker fan structure in terms of matroid subdivisions.

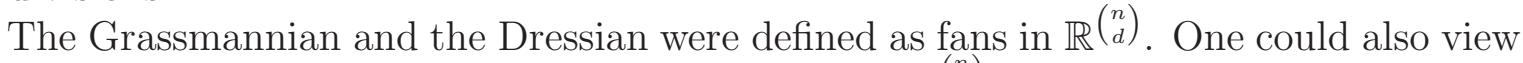
them as subcomplexes in the tropical projective space $\mathbb{T P}\left(\begin{array}{l}n \\ d\end{array}\right)-1$, which is the compact space

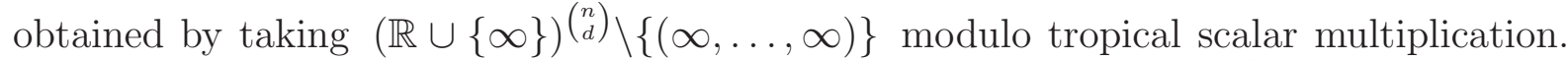

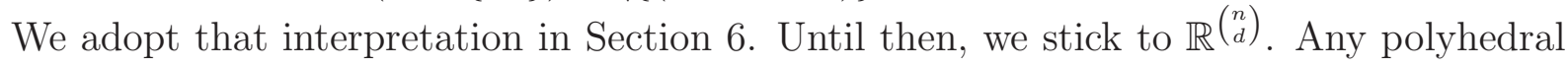
fan gives rise to an underlying (spherical) polytopal complex obtained by intersecting with the corresponding unit sphere. Moreover, the Grassmannian $\operatorname{Gr}(d, n)$ and the Dressian $\operatorname{Dr}(d, n)$ have the same $n$-dimensional lineality space which we can factor out. This gives pointed fans in $\mathbb{R}^{\left(\begin{array}{l}n \\ d\end{array}\right)-n}$. For the underlying spherical polytopal complexes of these pointed fans we again use the notation $\operatorname{Gr}(d, n)$ and $\operatorname{Dr}(d, n)$. The former has dimension 
$d(n-d)-n$, while the latter is a generally higher-dimensional polyhedral complex whose support contains the support of $\operatorname{Gr}(d, n)$. For instance, $\operatorname{Gr}(2,5)=\operatorname{Dr}(2,5)$ is the Petersen graph. In the sequel we will discuss topological features of $\operatorname{Gr}(d, n)$ and $\operatorname{Dr}(d, n)$. In these cases we always refer to the underlying polytopal complexes of these two fans modulo their lineality spaces. Each of the two fans is a cone over the underlying polytopal complex (joined with the lineality space). Hence the fans are topologically trivial, while the underlying polytopal complexes are not.

It is clear from the definitions that the Dressian contains the Grassmannian (over any

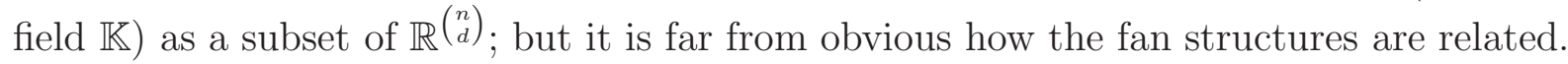
Results of [29] imply that $\operatorname{Gr}(2, n)=\operatorname{Dr}(2, n)$ as fans and that $\operatorname{Gr}(3,6)=\operatorname{Dr}(3,6)$ as sets. Using computations with the software systems Gfan [18], homology [10], Macaulay2 [19], and polymake [13] we obtained the following results about the next case $(d, n)=(3,7)$.

Theorem 2.1. Fix any field $\mathbb{K}$ of characteristic different from 2. The tropical Grassmannian $\operatorname{Gr}(3,7)$, with its induced Gröbner fan structure, is a simplicial fan with $f$-vector

$$
(721,16800,124180,386155,522585,252000) \text {. }
$$

The homology of the underlying five-dimensional simplicial complex is free Abelian, and it is concentrated in top dimension:

$$
H_{*}(\operatorname{Gr}(3,7) ; \mathbb{Z})=H_{5}(\operatorname{Gr}(3,7) ; \mathbb{Z})=\mathbb{Z}^{7470} .
$$

The result on the homology is consistent with Hacking's theorem in [15, Theorem 2.5]. Indeed, Hacking showed that if the tropical compactification is schön then the homology of the tropical variety is concentrated in top dimension, and it is conjectured in $[21, \S 1.4]$ that the property of being schön holds for the Grassmannian when $d=3$ and $n=7$; see also [15, Example 4.2]. Inspired by Markwig and Yu [22], we conjecture that the simplicial complex $\operatorname{Gr}(3,7)$ is shellable.

Theorem 2.2. The Dressian $\operatorname{Dr}(3,7)$, with its Plücker fan structure, is a non-simplicial fan. The underlying polyhedral complex is six-dimensional and has the f-vector

$$
(616,13860,101185,315070,431025,211365,30) \text {. }
$$

Its 5-skeleton is triangulated by the Grassmannian $\operatorname{Gr}(3,7)$, and the homology is

$$
H_{*}(\operatorname{Dr}(3,7) ; \mathbb{Z})=H_{5}(\operatorname{Dr}(3,7) ; \mathbb{Z})=\mathbb{Z}^{7440} .
$$

We note that the combinatorial and algebraic notions in this paper are compatible with the geometric theory developed in Mikhalkin's book [23]. We here use "min" for tropical addition, the set $\mathbb{T}^{k-1}=\mathbb{R}^{k} / \mathbb{R}(1,1, \ldots, 1)$ is the tropical torus, and the tropical projective space $\mathbb{T P}^{k-1}$ is a compactification of $\mathbb{T}^{k-1}$ which is a closed simplex.

The symmetric group $\mathrm{S}_{7}$ acts naturally on both $\operatorname{Gr}(3,7)$ and $\operatorname{Dr}(3,7)$, and it makes sense to count their cells up to this symmetry. The face numbers of the underlying polytopal complexes modulo $\mathrm{S}_{7}$ are

$$
\begin{aligned}
& f\left(\operatorname{Gr}(3,7) \bmod \mathrm{S}_{7}\right)=(6,37,140,296,300,125) \text { and } \\
& f\left(\operatorname{Dr}(3,7) \bmod \mathrm{S}_{7}\right)=(5,30,107,217,218,94,1) .
\end{aligned}
$$


Thus the Grassmannian $\operatorname{Gr}(3,7)$ modulo $\mathrm{S}_{7}$ has 125 five-dimensional simplices, and these are merged to 94 five-dimensional polytopes in the Dressian $\operatorname{Dr}(3,7)$ modulo $\mathrm{S}_{7}$. One of these cells is not a facet because it lies in the unique cell of dimension six. This means that $\operatorname{Dr}(3,7)$ has $93+1=94$ facets (= maximal cells) up to the $\mathrm{S}_{7}$-symmetry.

Each point in $\operatorname{Dr}(3, n)$ determines a plane in $\mathbb{T} \mathbb{P}^{n-1}$. This map was described in $[27,29]$ and we recall it in Section 5 . The cells of $\operatorname{Dr}(3, n)$ modulo $\mathrm{S}_{n}$ correspond to combinatorial types of tropical planes. Facets of $\operatorname{Dr}(3, n)$ correspond to generic planes in $\mathbb{T P}^{n-1}$ :

Corollary 2.3. The number of combinatorial types of generic planes in $\mathbb{T P}^{6}$ is 94 . The numbers of types of generic planes in $\mathbb{T P}^{3}, \mathbb{T P}^{4}$, and $\mathbb{T} \mathbb{P}^{5}$ are 1,1 , and 7 , respectively.

Proof. The unique generic plane in $\mathbb{T P}^{3}$ is the cone over the complete graph $K_{4}$. Planes in $\mathbb{T P}^{4}$ are parameterized by the Petersen graph $\operatorname{Dr}(3,5)=\operatorname{Gr}(3,5)$, and the unique generic type is dual to the trivalent tree with five leaves. The seven types of generic planes in $\mathbb{T P}^{5}$ were derived in $[29, \S 5]$. Drawings of their bounded parts are given in Figure 1, while their unbounded cells are represented by the tree arrangements in Table 2 below. The number 94 for $n=7$ is derived from Theorem 2.2.

A complete census of all combinatorial types of tropical planes in $\mathbb{T P}^{6}$ is posted at Www .uni-math.gwdg.de/jensen/Research/G3_7/grassmann3_7.html.

This web site and the notation used therein is a main contribution of the present paper. In the rest of this section we explain how our two classification theorems were obtained.

Computational proof of Theorem 2.1. The Grassmannian $\operatorname{Gr}(3,7)$ is the tropical variety defined by the Plücker ideal $I_{3,7}$ in the polynomial ring $\mathbb{K}\left[p_{S}\right]$ in 35 unknowns. We first suppose that $\mathbb{K}$ has characteristic zero, and for our computations we take $\mathbb{K}=\mathbb{Q}$. The subvariety of $\mathbb{P}_{\mathbb{Q}}^{34}$ defined by $I_{3,7}$ is irreducible of dimension 12 and has an effective sixdimensional torus action. The Bieri-Groves Theorem [4] ensures that $\operatorname{Gr}(3,7)$ is a pure five-dimensional subcomplex of the Gröbner complex of $I_{3,7}$. Moreover, by [6, Theorem 3.1], this complex is connected in codimension one. The software Gfan [18] exploits this connectivity by traversing the facets exhaustively when computing $\operatorname{Gr}(3,7)=\mathbb{T}\left(I_{3,7}\right)$.

The input to Gfan is a single maximal Gröbner cone of the tropical variety. The cone is, as described in the Gfan manual, represented by a pair of Gröbner bases. Knowing a relative interior point of a maximal cone we can compute this pair with the command

gfan_initialforms --ideal --pair

run on the input

$\mathrm{Q}$ [p123,p124,p125,p126,p127,p134,p135,p136,p137,p145,p146,p147, p156,p157, p167,p234, p235, p236 ,p237,p245, p246, p247, p256 ,p257,p267, p345, p346, p347, p356, p357, p367,p456,p457,p467,p567]

\{

p123*p145-p124*p135+p125*p134,

... .

THE ELECTRONiC Journal of COMBINATORICs 16(2) (2009), \#R6 


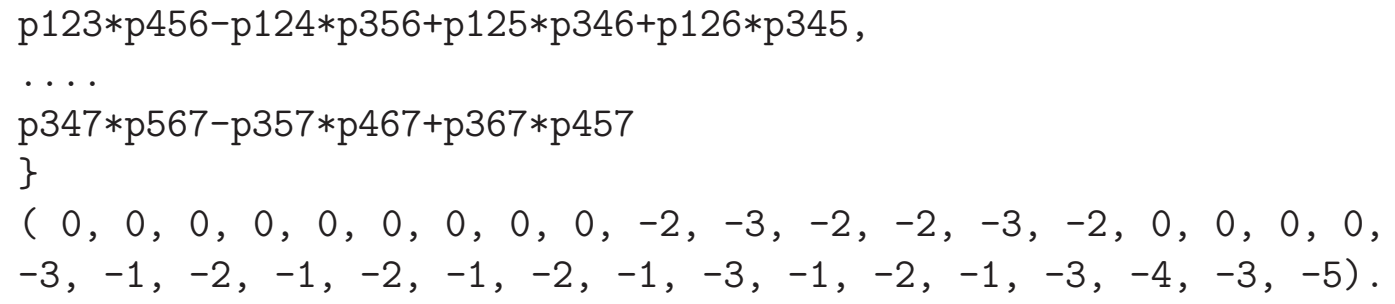

The polynomials are the 140 quadrics which minimally generate the Plücker ideal $I_{3,7}$. Among these are 105 three-term relations and 35 four-term relations. Since Gfan uses the max-convention for tropical addition, weight vectors have to be negated. The output is handed over to the program gfan_tropicaltraverse, which computes all other maximal cones. For this computation to finish it is decisive to use the --symmetry option. The symmetric group $S_{7}$ acts on the tropical Plücker coordinates as a subgroup of $S_{35}$. In terms of classical Plücker coordinates, these symmetries only exist if we simultaneously perform sign changes, such as $p_{132}=-p_{123}$. We inform Gfan about these sign changes using --symsigns, and we specify the sign changes on the input as elements of $\{-1,+1\}^{35}$ together with the generators of $\mathrm{S}_{7} \subset \mathrm{S}_{35}$ after the Gröbner basis pair produced above:

$\{(15,16,17,18,0,19,20,21,1,22,23,2,24,3,4,25,26,27,5,28,29,6,30,7,8,31$, $32,9,33,10,11,34,12,13,14),(0,1,2,3,4,15,16,17,18,19,20,21,22,23,24,5$, $6,7,8,9,10,11,12,13,14,25,26,27,28,29,30,31,32,33,34)\}$ $\{(1,1,1,1,1,1,1,1,1,1,1,1,1,1,1,1,1,1,1,1,1,1,1,1,1,1,1,1,1,1,1,1,1,1,1)$, $(-1,-1,-1,-1,-1,1,1,1,1,1,1,1,1,1,1,1,1,1,1,1,1,1,1,1,1,1,1,1,1,1,1,1,1$, $1,1)\}$

Before traversing $\operatorname{Gr}(3,7)$, Gfan verifies algebraically that these indeed are symmetries.

In order to handle a tropical variety as large as $\operatorname{Gr}(3,7)$, the implementation of the traversal algorithm in [6] was improved in several ways. During the traversal of the maximal cones up to symmetry, algebraic tests were translated into polyhedral containment questions whenever possible. Since the fan turned out to be simplicial, computing the rays could be reduced to linear algebra while in general Gfan uses the double description method of cddlib [12]. In the subsequent combinatorial extraction of all faces up to symmetry, checking if two cones are in the same orbit can be done at the level of canonical interior points. Checking if two points are equal up to symmetry was done by running through all permutations in the group. This may not be optimal but is sufficient for our purpose. For further speed-ups we linked Gfan to the floating point LP solver SoPlex [32] which produced certificates verifiable in integer arithmetic. In case of a failure caused by round-off errors, the program falls back on cddlib which solves the LP problem in exact arithmetic. The running time for the computation is approximately 25 hours on a standard desktop computer with Gfan version 0.4, which will be released by May 2009 . The output of Gfan is in polymake [13] format, and the program homology [10] was used to compute the integral homology of the underlying polytopal complex.

The above computations established our result in characteristic zero. To obtain the same result for prime characteristics $p \geq 3$, we used Macaulay2 to redo all Gröbner basis computations, one for each cone in $\operatorname{Gr}(3,7)$, in the polynomial ring $\mathbb{Z}\left[p_{S}\right]$ over the integers. 
We found that all but one of the initial ideals $\operatorname{in}_{\lambda}\left(I_{3,6}\right)$ arise from $I_{3,6}$ via a Gröbner basis whose coefficients are +1 and -1 . Hence these cones of $\operatorname{Gr}(3,7)$ are characteristic-free. The only exception is the Fano cone which will be discussed in the end of Section 3.

Computational proof of Theorem 2.2. For $d=3$ and $n=7$ there are 105 three-term Plücker relations (1). A vector $\lambda \in \mathbb{R}^{35}$ lies in $\operatorname{Dr}(3,7)$ if and only if the initial form of each three-term relation with respect to $\lambda$ has either two or three terms. There are four possibilities for this to happen, and each choice is described by a linear system of equations and inequalities. This system is feasible if and only if the corresponding cone exists in the Dressian $\operatorname{Dr}(3,7)$, and this can be tested using linear programming. In theory, we could compute the Dressian by running a loop over all $4^{105}$ choices and list which choices determine a non-empty cone of $\operatorname{Dr}(3,7)$. Clearly, this is infeasible in practice.

To control the combinatorial explosion, we employed the representation of tropical planes by abstract tree arrangements which will be introduced in Section 4. This representation allows a recursive computation of $\operatorname{Dr}(3, n)$ from $\operatorname{Dr}(3, n-1)$. The idea is similar to what is described in the previous paragraph, but the approach is much more efficient. By taking the action of the symmetric group of degree $n$ into account and by organizing this exhaustive search well enough this leads to a viable computation. A key issue seems to be to focus on the equations early in the enumeration, while the inequalities are considered only at the very end. A polymake implementation enumerates all cones of $\operatorname{Dr}(3,7)$ within one hour. The same computation for $\operatorname{Dr}(3,6)$ takes less than two minutes.

Again we used homology for computing the integral homology of the underlying polytopal complex of $\operatorname{Dr}(3,7)$. Since $\operatorname{Dr}(3,7)$ is not simplicial it cannot be fed into homology directly. However, it is homotopy equivalent to its crosscut complex, which thus has the same homology [5]. The crosscut complex (with respect to the atoms) is the abstract simplicial complex whose vertices are the rays of $\operatorname{Dr}(3,7)$ and whose faces are the subsets of rays which are contained in cones of $\operatorname{Dr}(3,7)$. The computation of the homology of the crosscut complex takes about two hours.

Remark 2.4. Following [8,9], a valuated matroid of rank $d$ on the set $[n]$ is a map $\pi:[n]^{d} \rightarrow \mathbb{R} \cup\{\infty\}$ such that $\pi(\omega)$ is independent of the ordering of the sequence $\omega$, $\pi(\omega)=\infty$ if an element occurs twice in $\omega$, and the following axiom holds: for every $(d-1)$-subset $\sigma$ and every $(d+1)$-subset $\tau=\left\{\tau_{1}, \tau_{2}, \ldots, \tau_{d+1}\right\}$ of $[n]$ the minimum of

$$
\pi\left(\sigma \cup\left\{\tau_{i}\right\}\right)+\pi\left(\tau \backslash\left\{\tau_{i}\right\}\right) \quad \text { for } \quad 1 \leq i \leq d+1
$$

is attained at least twice. Results of Dress and Wenzel [8] imply that tropical Plücker vectors and valuated matroids are the same. To see this, one applies [8, Theorem 3.4] to the perfect fuzzy ring arising from $(\mathbb{R} \cup\{\infty\}, \min ,+)$ via the construction in [8, page 182].

\section{Matroid Subdivisions}

A weight function $\lambda$ on an $n$-dimensional polytope $P$ in $\mathbb{R}^{n}$ assigns a real number to each vertex of $P$. The lower facets of the lifted polytope $\operatorname{conv}\{(v, \lambda(v)) \mid v$ vertex of $P\}$ 
in $\mathbb{R}^{n+1}$ induce a polytopal subdivision of $P$. Polytopal subdivisions arising in this way are called regular. The set of all weights inducing a fixed subdivision forms a (relatively open) polyhedral cone, and the set of all these cones is a complete fan, the secondary fan of $P$. The dimension of the secondary fan as a spherical complex is $m-n-1$, where $m$ is the number of vertices of $P$. For a detailed introduction to these concepts see [7].

We denote the canonical basis vectors of $\mathbb{R}^{n}$ by $e_{1}, e_{2}, \ldots, e_{n}$, and we abbreviate $e_{X}:=$ $\sum_{i \in X} e_{i}$ for any subset $X \subseteq[n]$. For a set $X \subseteq\left(\begin{array}{c}{[n]} \\ d\end{array}\right)$ we define the polytope

$$
P_{x}:=\operatorname{conv}\left\{e_{X} \mid X \in X\right\} .
$$

The $d$-th hypersimplex in $\mathbb{R}^{n}$ is the special case

$$
\Delta(d, n):=P_{\left(\begin{array}{c}
{[n]} \\
d
\end{array}\right)} .
$$

A subset $\mathcal{M} \subseteq\left(\begin{array}{c}{[n]} \\ d\end{array}\right)$ is a matroid of rank $d$ on the set $[n]$ if the edges of the polytope $P_{\mathcal{M}}$ are all parallel to the edges of $\Delta(d, n)$; in this case $P_{\mathcal{M}}$ is called a matroid polytope, and the elements of $\mathcal{M}$ are the bases. That this definition really describes a matroid as, for example, in White [31], is a result of Gel'fand, Goresky, MacPherson, and Serganova [14]. Moreover, each face of a matroid polytope is again a matroid polytope [11]. A polytopal subdivision of $\Delta(d, n)$ is a matroid subdivision if each of its cells is a matroid polytope.

Proposition 3.1. (Speyer [27, Proposition 2.2]) A weight vector $\lambda \in \mathbb{R}^{\left(\begin{array}{c}{[n]} \\ d\end{array}\right)}$ lies in the Dressian $\operatorname{Dr}(d, n)$, seen as a fan, if and only if it induces a matroid subdivision of the hypersimplex $\Delta(d, n)$.

The weight functions inducing matroid subdivisions form a subfan of the secondary fan of $\Delta(d, n)$, and this defines the secondary fan structure on the Dressian $\operatorname{Dr}(d, n)$. It is not obvious whether the secondary fan structure and the Plücker fan structure on $\operatorname{Dr}(d, n)$ coincide. We shall see in Theorem 4.4 that this is indeed the case for $d=3$. In particular, the rays of the Dressian $\operatorname{Dr}(3, n)$ correspond to coarsest matroid subdivisions of $\Delta(3, n)$.

Corollary 3.2. Let $M$ be a connected matroid of rank $d$ on $[n]$ and let $\lambda_{M} \in\{0,1\}\left(\begin{array}{c}{[n]} \\ d\end{array}\right)$ be the vector which satisfies $\lambda_{M}(X)=0$ if $X$ is a basis of $M$ and $\lambda_{M}(X)=1$ if $X$ is not a basis of $M$. Then $\lambda_{M}$ lies in the Dressian $\operatorname{Dr}(d, n)$, and the corresponding matroid decomposition of $\Delta(d, n)$ has the matroid polytope $P_{M}$ as a maximal cell.

Proof. The basis exchange axiom for matroids translates into a combinatorial version of the quadratic Plücker relations (cf. Remark 2.4), and this ensures that the vector $\lambda_{M}$ lies in the Dressian $\operatorname{Dr}(d, n)$. By Proposition 3.1, the regular subdivision of $\Delta(d, n)$ defined by $\lambda_{M}$ is a matroid subdivision. The matroid polytope $P_{M}$ appears as a lower face in the lifting of $\Delta(d, n)$ by $\lambda_{M}$, and hence it is a cell of the matroid subdivision. It is a maximal cell because $\operatorname{dim}\left(P_{M}\right)=n-1$ if and only if the matroid $M$ is connected; see [11].

Each vertex figure of $\Delta(d, n)$ is isomorphic to the product of simplices $\Delta_{d-1} \times \Delta_{n-d-1}$. A regular subdivision of a polytope induces regular subdivisions on its facets as well as on its vertex figures. For hypersimplices the converse holds (see also Proposition 4.5): 
Proposition 3.3. (Kapranov [20, Corollary 1.4.14]). Each regular subdivision of the product of simplices $\Delta_{d-1} \times \Delta_{n-d-1}$ is induced by a regular matroid subdivision of $\Delta(d, n)$.

A split of a polytope is a regular subdivision with exactly two maximal cells. By [17, Lemma 7.4], every split of $\Delta(d, n)$ is a matroid subdivision. Collections of splits that are pairwise compatible define a simplicial complex, known as the split complex of $\Delta(d, n)$. It was shown in [17, Section 7] that the regular subdivision defined by pairwise compatible splits is always a matroid subdivision. The following result appears in [17, Theorem 7.8]:

Proposition 3.4. The split complex of $\Delta(d, n)$ is a simplicial subcomplex of the Dressian $\operatorname{Dr}(d, n)$, with its secondary complex structure. They are equal if $d=2$ or $d=n-2$.

Special examples of splits come about in the following way. The vertices adjacent to a fixed vertex of $\Delta(d, n)$ span a hyperplane which defines a split; and these splits are called vertex splits. Moreover, two vertex splits are compatible if and only if the corresponding vertices of $\Delta(d, n)$ are not connected by an edge. Hence the simplicial complex of stable sets of the edge graph of $\Delta(d, n)$ is contained in the split complex of $\Delta(d, n)$.

Corollary 3.5. The simplicial complex of stable sets of the edge graph of the hypersimplex $\Delta(d, n)$ is a subcomplex of $\operatorname{Dr}(d, n)$. Hence, the dimension of the Dressian $\operatorname{Dr}(d, n)$, seen as a polytopal complex, is bounded below by one less than the maximal size of a stable set of this edge graph.

We shall use this corollary to prove the main result in this section. Recall that the dimension of the Grassmannian $\operatorname{Gr}(3, n)$ equals $2 n-9$. Consequently, the following theorem implies that, for large $n$, most of the tropical planes (cf. Section 5) are not realizable.

Theorem 3.6. The dimension of the Dressian $\operatorname{Dr}(3, n)$ is of order $\Theta\left(n^{2}\right)$.

For the proof of this result we need one more definition. The spread of a vector in $\operatorname{Dr}(d, n)$ is the number of maximal cells of the corresponding matroid decomposition. The splits are precisely the vectors of spread 2, and these are rays of $\operatorname{Dr}(d, n)$, seen as a fan. The rays of $\operatorname{Dr}(3,6)$ are either of spread 2 or 3 ; see $[29, \S 5]$. As a result of our computation the spreads of rays of $\operatorname{Dr}(3,7)$ turn out to be 2,3 , and 4 . We note the following result.

Proposition 3.7. As $n$ increases, the spread of the rays of $\operatorname{Dr}(3, n)$ is not bounded by a constant.

Proof. By Proposition 3.3, each regular subdivision of $\Delta_{2} \times \Delta_{n-4}$ is induced by a regular matroid subdivision of $\Delta(3, n)$, and hence, in light of the Cayley trick [26], by mixed subdivisions of the dilated triangle $(n-3) \Delta_{2}$. See also Section 4. This correspondence maps rays of the secondary fan of $\Delta_{2} \times \Delta_{n-4}$ to rays of the $\operatorname{Dressian} \operatorname{Dr}(3, n)$. Now, a coarsest mixed subdivision of $(n-3) \Delta_{2}$ can have arbitrarily many polygons as $n$ grows large. For an example consider the hexagonal subdivision in [26, Figure 12]. Hence a coarsest regular matroid subdivision of $\Delta(3, n)$ can have arbitrarily many facets. 
Proof of Theorem 3.6. Speyer [27, Theorem 6.1] showed that the spread of any vector in $\operatorname{Dr}(d, n)$ is at most $\left(\begin{array}{l}n-2 \\ d-1\end{array}\right)$. This is the maximal number of facets of any matroid subdivision of $\Delta(d, n)$. Consider a flag of faces $F_{1} \subset F_{2} \subset \cdots$ in the underlying polytopal complex of $\operatorname{Dr}(d, n)$. For every $i$ the subdivision corresponding to $F_{i}$ has more facets than that of $F_{i-1}$. Hence $\left(\begin{array}{l}n-2 \\ d-1\end{array}\right)-1$ is an upper bound for the dimension of $\operatorname{Dr}(d, n)$. Specializing to $d=3$, this upper bound is quadratic.

We shall apply Proposition 3.4 to derive the lower bound. The generalized Fano matroid $\mathcal{F}_{r}$ is a connected simple matroid on $2^{r}-1$ points which has rank 3 and is defined as follows. Its three-element circuits are the lines of the $(r-1)$-dimensional projective space $\mathrm{PG}_{r-1}(2)$ over the field $\mathbb{G} \mathbb{F}(2)$ with two elements. The total number of unordered bases of $\mathcal{F}_{r}$, that is, non-collinear triples of points, equals

$$
\beta_{r}:=\frac{1}{6}\left(2^{r}-1\right)\left(2^{r}-2\right)\left(2^{r}-4\right)
$$

The number of vertices of $\Delta\left(3,2^{r}-1\right)$ which are not bases of $\mathcal{F}_{r}$ equals

$$
\nu_{r}:=\left(\begin{array}{c}
2^{r}-1 \\
3
\end{array}\right)-\beta_{r}=\frac{1}{6}\left(2^{r}-1\right)\left(2^{r}-2\right) .
$$

We claim that the non-bases of $\mathcal{F}_{r}$ form a stable set in the edge graph of $\Delta\left(3,2^{r}-1\right)$. Indeed, the non-bases are precisely the collinear triplets of points, that is, the full point rows of the lines in $\mathrm{PG}_{r-1}(2)$. Two distinct point rows of lines in $\mathrm{PG}_{r-1}(2)$ share at most one point, and hence the two corresponding vertices of $\Delta\left(3,2^{r}-1\right)$ do not differ by an exchange of two bits, which means that they are not connected by an edge.

The quadratic lower bound is now derived from Proposition 3.4 as follows. For given any $n$, let $r$ be the unique natural number satisfying $2^{r}-1 \leq n<2^{r+1}$. Then the generalized Fano matroid $\mathcal{F}_{r}$ yields a stable set of size $\nu_{r}=1 / 6\left(2^{r}-1\right)\left(2^{r}-2\right) \geq n^{2} / 24-$ $n / 12$ in the edge graph of $\Delta(3, n)$. The latter inequality follows from $2^{r}-1 \geq n / 2$.
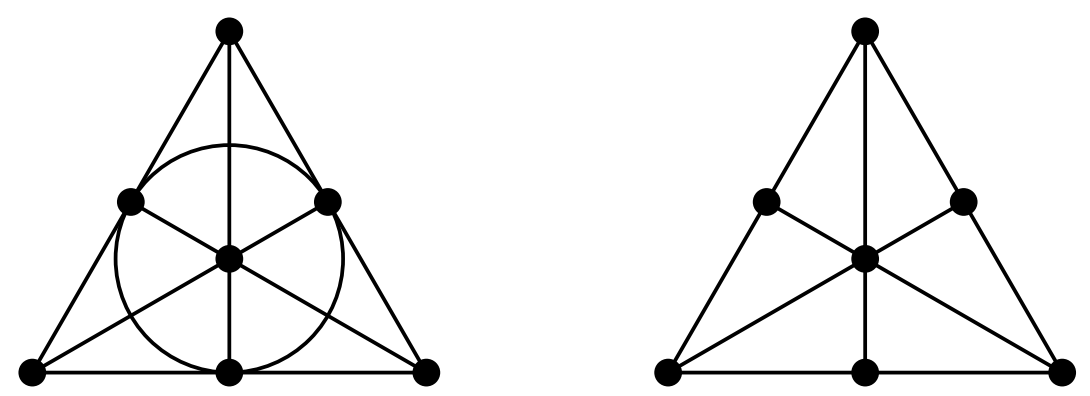

Figure 2: The point configurations for the Fano and non-Fano matroids.

Computational proof of Theorem 2.1 (continued). We still have to discuss the Fano cone of $\operatorname{Dr}(3,7)$ and its relationship to $\operatorname{Gr}(3,7)$. The matroid $\mathcal{F}_{3}$ in the proof of Theorem 3.6 corresponds to the Fano plane $\mathrm{PG}_{2}(2)$, which is shown in Figure 2 on the left. We have 
$\beta_{3}=28$ and $\nu_{3}=7$. Via Corollary 3.2 the Fano matroid $\mathcal{F}_{3}$ gives rise to a cone in the fan $\operatorname{Dr}(3,7)$ which we call the Fano cone. The corresponding cell of $\operatorname{Dr}(3,7)$, seen as a polytopal complex, has dimension six. Moreover, all 30 six-dimensional cells of $\operatorname{Dr}(3,7)$ come from the Fano matroid $\mathcal{F}_{3}$ by relabeling. They form a single orbit under the $\mathrm{S}_{7}$ action, since the automorphism group $\mathrm{GL}_{3}(2)$ of $\mathcal{F}_{3}$ has order $168=5040 / 30$. If the field $\mathbb{K}$ considered has characteristic 2 then the Fano cell of $\operatorname{Dr}(3,7)$ intersects $\operatorname{Gr}(3,7)$ in a five-dimensional complex that looks like a tropical hyperplane.

Finally, suppose that the characteristic of $\mathbb{K}$ is different from 2. Since the Fano matroid is not realizable over $\mathbb{K}$, the Fano cone of $\operatorname{Dr}(3,7)$ corresponds to a non-realizable tropical plane in $\mathbb{T P}^{6}$ and the intersection of the Fano cell with $\operatorname{Gr}(3,7)$ is a five-dimensional simplicial sphere arising from seven copies of the non-Fano matroid; see Figure 2 on the right. In this case this also gives us the difference in the homology of $\operatorname{Dr}(3,7)$ and $\operatorname{Gr}(3,7)$. The Fano six-cells are simplices. Each of them cancels precisely one homology cycle of $\operatorname{Gr}(3,7)$.

In spite of the results in this sections, many open problems remain. Here are two specific questions we have concerning the combinatorial structure of the Dressian $\operatorname{Dr}(3, n)$ :

$\triangleright$ Are all rays of $\operatorname{Dr}(3, n)$ always rays of $\operatorname{Gr}(3, n)$ ?

$\triangleright$ Characterize the rays of $\operatorname{Dr}(3, n)$, that is, coarsest matroid subdivisions of $\Delta(3, n)$.

\section{Tree Arrangements}

Let $n \geq 4$ and consider an $n$-tuple of metric trees $T=\left(T_{1}, T_{2}, \ldots, T_{n}\right)$ where $T_{i}$ has the set of leaves $[n] \backslash\{i\}$. A metric tree $T_{i}$ by definition comes with non-negative edge lengths, and by adding lengths along paths it defines a metric $\delta_{i}:([n] \backslash\{i\}) \times([n] \backslash\{i\}) \rightarrow \mathbb{R}_{\geq 0}$. We call the $n$-tuple $T$ of metric trees a metric tree arrangement if

$$
\delta_{i}(j, k)=\delta_{j}(k, i)=\delta_{k}(i, j)
$$

for all $i, j, k \in[n]$ pairwise distinct. Moreover, considering trees $T_{i}$ without metrics, but with leaves still labeled by $[n] \backslash\{i\}$, we say that $T$ is an abstract tree arrangement if

$\triangleright$ either $n=4$;

$\triangleright$ or $n=5$, and $T$ is the set of quartets of a tree with five leaves;

$\triangleright$ or $n \geq 6$, and $\left(T_{1} \backslash i, \ldots, T_{i-1} \backslash i, T_{i+1} \backslash i, \ldots, T_{n} \backslash i\right)$ is an arrangement of $n-1$ trees for each $i \in[n]$.

Here $T_{j} \backslash i$ denotes the tree on $[n] \backslash\{i, j\}$ gotten by deleting leaf $i$ from tree $T_{j}$. A quartet of a tree is a subtree induced by four of its leaves.

The following result relates the two concepts of tree arrangements we introduced: 
Proposition 4.1. Each metric tree arrangement gives rise to an abstract tree arrangement by ignoring the edge lengths. The converse is not true: for $n \geq 9$, there exist abstract arrangements of $n$ trees that do not support any metric tree arrangement.

Proof. The first assertion follows from the Four Point Condition; see [24, Theorem 2.36]. An example establishing the second assertion is the abstract arrangement of nine trees listed in Table 1 and depicted in Figure 3: Three of the trees (numbered 1,2,3) are on the boundary, while the six remaining trees (numbered 4, 5,6,7,8,9) partition the dual graph of the subdivision of the big triangle into quadrangles and small triangles. Each intersection of the tree $T_{a}$ with the tree $T_{b}$ in one of the quadrangles defines a leaf labeled $b$ in $T_{a}$ and, symmetrically, a leaf labeled $a$ in $T_{b}$. See Example 4.7 below for more details, including an argument why this abstract arrangement cannot be realized as a metric arrangement.

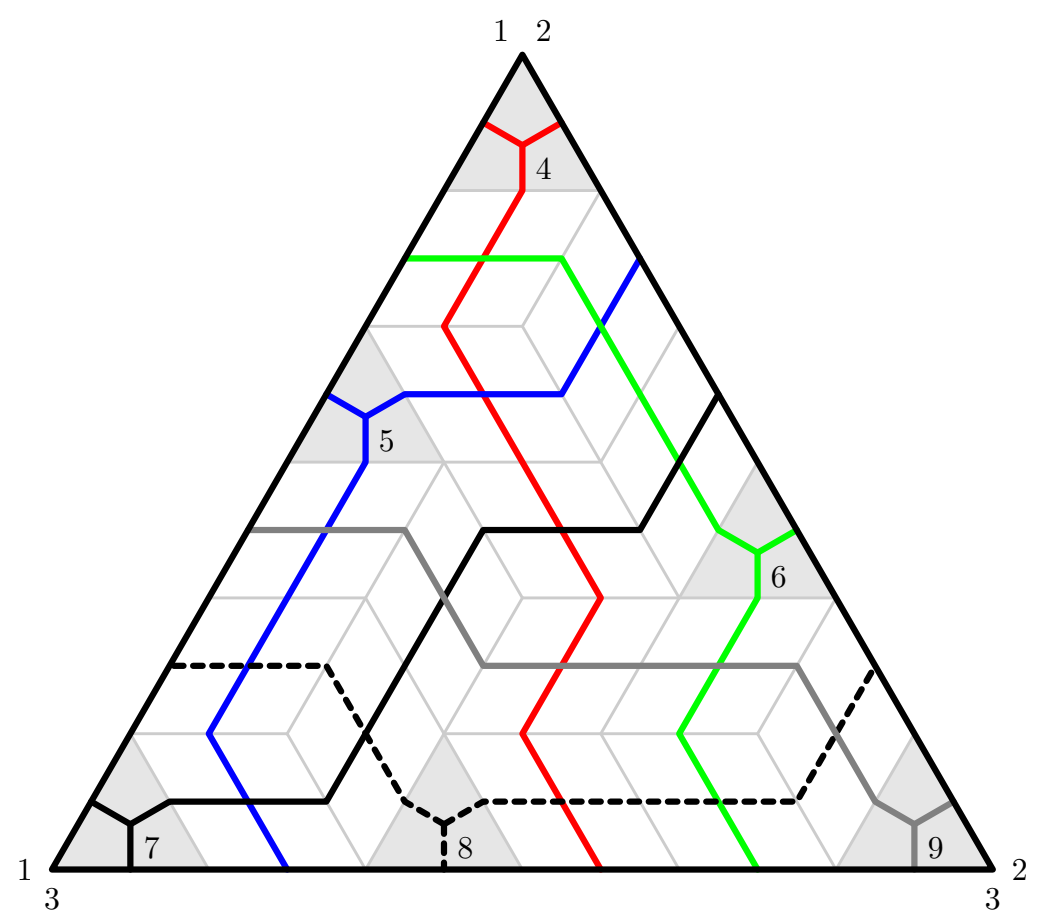

Figure 3: Abstract arrangement of nine caterpillar trees on eight leaves encoding a matroid subdivision of $\Delta(3,9)$ which is not regular; see Table 1.

The hypersimplex $\Delta(d, n)$ is the intersection of the unit cube $[0,1]^{n}$ with the affine hyperplane $\sum x_{i}=d$. From this it follows that the facets of $\Delta(d, n)$ correspond to the facets of $[0,1]^{n}$. We call the facet defined by $x_{i}=0$ the $i$-th deletion facet of $\Delta(d, n)$, and the facet defined by $x_{i}=1$ the $i$-th contraction facet. These names come about as follows: If $\mathcal{M}$ is a matroid on $[n]$ of rank $d$ then the intersection of $P_{\mathcal{M}}$ with the $i$-th deletion (contraction) facet is the matroid polytope of the matroid obtained by deleting (contracting) $i$. Each deletion facet of $\Delta(d, n)$ is isomorphic to $\Delta(d, n-1)$, and each contraction facet is isomorphic to $\Delta(d-1, n-1)$. We use the terms "deletion" and 


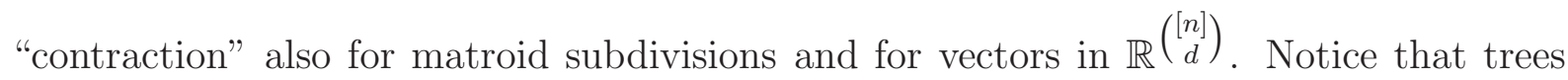
come naturally into the game since a polytopal subdivision of $\Delta(2, n-1)$ is a matroid subdivision if and only if it is dual to a tree.

Lemma 4.2. Each matroid subdivision $\Sigma$ of $\Delta(3, n)$ defines an abstract arrangement $T(\Sigma)$ of $n$ trees. Moreover, if $\Sigma$ is regular then $T(\Sigma)$ supports a metric tree arrangement.

Proof. Each of the $n$ contraction facets of $\Delta(3, n)$ is isomorphic to $\Delta(2, n-1)$, and thus $\Sigma$ induces matroid subdivisions on $n$ copies of $\Delta(2, n-1)$. But the matroid subdivisions of $\Delta(2, n-1)$ are generated by compatible systems of splits, see [29, Theorem 3.4] and $[17, \S 6]$. These matroid subdivisions are dual to trees, and hence $\Sigma$ gives rise to a tree arrangement.

Now let $\Sigma$ be regular with weight function $\lambda$. By adding or subtracting a suitable multiple of $(1,1, \ldots, 1)$ and subsequent rescaling we can assume that $\lambda$ attains values between 1 and 2 only. The function $\lambda$ can be restricted to a weight function on each contraction facet. But a weight function on $\Delta(2, n-1)$ which takes values between 1 and 2 is a metric. Since the induced regular subdivisions of the facets of $\Delta(3, n)$ isomorphic to $\Delta(2, n-1)$ are also regular matroid subdivisions, they are dual to metric trees with $n-1$ leaves. The Split Decomposition Theorem of Bandelt and Dress [3, Theorem 2] allows to read off the lengths on all edges of these trees; see also [17, Theorem 3.10].

Proposition 4.3. Let $\Sigma$ and $\bar{\Sigma}$ be two matroid subdivisions of $\Delta(3, n)$ such that $\Sigma$ refines $\bar{\Sigma}$. If $\Sigma$ and $\bar{\Sigma}$ induce the same subdivision on the boundary of $\Delta(3, n)$ then $\Sigma$ and $\bar{\Sigma}$ coincide.

Proof. Suppose that $\Sigma$ strictly refines $\bar{\Sigma}$. Then there is a codimension-1-cell $F$ of $\Sigma$ which is not a cell in $\bar{\Sigma}$. Let $\bar{F}$ be the unique full-dimensional cell of $\bar{\Sigma}$ that contains $F$. In particular, $F$ is not contained in the boundary of $\Delta(3, n)$. Then $F$ is a rank-3-matroid polytope $F=P_{\mathcal{M}}$ of codimension 1 where $\mathcal{M}=\mathcal{M}_{1} \cup \mathcal{M}_{2}$ is the disjoint union of a rank1-matroid $\mathcal{M}_{1}$ and a rank-2-matroid $\mathcal{M}_{2}$. In particular, $F \cong P_{\mathcal{M}_{1}} \times P_{\mathcal{M}_{2}}$. Notice that the affine hull $H$ of $F$ is defined by the equation $\sum_{i \in I} x_{i}=1$ where we denote by $I$ the set of elements of $\mathcal{M}_{1}$, all of which are parallel because of $\operatorname{rank} \mathcal{M}_{1}=1$.

Since $\bar{F}$ is subdivided by $H$ there exist vertices $v, w$ of $\bar{F}$ on either side of $H$. Now $\bar{F}$ is also a matroid polytope of some matroid $\overline{\mathcal{M}}$ containing $\mathcal{M}$ as a submatroid. Up to relabeling we can assume that $v=e_{12 i}$ and $w=e_{345}$ such that $\{1,2, i\}$ and $\{3,4,5\}$ are bases of $\overline{\mathcal{M}}$ which are not bases of $\mathcal{M}$ and where $1,2 \in I$ and $i, 3,4,5 \notin I$. If $i \notin\{3,4,5\}$ we can exchange $i$ in the basis $\{1,2, i\}$ by some $j \in\{3,4,5\}$ to obtain a new basis of $\mathcal{\mathcal { M }}$. Without loss of generality we can assume that $i=5$ or $j=5$. Hence $\{1,2,5\}$ and $\{3,4,5\}$ are bases of $\overline{\mathcal{M}}$ that are not bases of $\mathcal{M}$. Notice that $e_{125}$ and $e_{345}$ are still on different sides of $H$ as $e_{12 i}$ and $e_{125}$ are connected by an edge and $\{1,2,5\}$ is not a basis of $\mathcal{M}$.

Now, as rank $\mathcal{M}_{i} \leq 2$, both $\mathcal{M}_{1}$ and $\mathcal{M}_{2}$ are realizable as affine point configurations over $\mathbb{R}$, and hence so is $\mathcal{M}$. In the sequel we identify $\mathcal{M}$ with a suitable point configuration (with multiple points). This way we obtain a subconfiguration of five points in $\mathcal{M}$ which looks like one of the two configurations shown in Figure 4. 


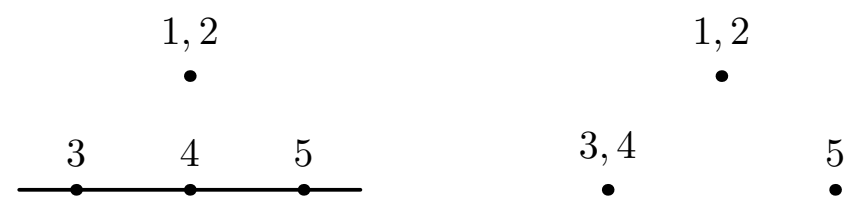

Figure 4: Two point configurations in the Euclidean plane.

Consider the intersection of $\Delta(3, n)$ with the affine space defined by $x_{5}=1$ and $x_{6}=x_{7}=\cdots=x_{n}=0$. This gives us an octahedron $C \cong \Delta(2,4)$ in the boundary of $\Delta(3, n)$. The intersection $S=F \cap C$ is a square; it can be read off Figure 4 as the convex hull of the four points $e_{135}, e_{145}, e_{235}$, and $e_{245}$. In particular, the square $S$ is a cell of $\Sigma$. However, since $e_{125}$ and $e_{345}$ are vertices of $\bar{F}=P_{\overline{\mathcal{M}}}$ as discussed above, $C$ is a cell of $\bar{\Sigma}$. We conclude that the square $S$ is a cell of $\Sigma$ but not a cell of $\bar{\Sigma}$. By construction $S \subset C$ is contained in the boundary of $\Delta(3, n)$. This yields the desired contradiction, as $\Sigma$ and $\bar{\Sigma}$ induce the same subdivision on the boundary.

Two metric tree arrangements are equivalent if they induce the same abstract tree arrangement. The following is the main result of this section.

Theorem 4.4. The equivalence classes of arrangements of $n$ metric trees are in bijection with the regular matroid subdivisions of the hypersimplex $\Delta(3, n)$. Moreover, the secondary fan structure on $\operatorname{Dr}(3, n)$ coincides with the Plücker fan structure.

Proof. Each regular matroid subdivision defines a metric tree arrangement by Lemma 4.2. The harder direction is to show that each metric tree arrangement gives rise to a regular matroid subdivision. We will prove this by induction on $n$. The hypersimplex $\Delta(3,4)$ is a 3 -simplex without any non-trivial subdivisions, and $\operatorname{Dr}(3,4)$ is a single point corresponding to the unique equivalence class of metric trees. The hypersimplex $\Delta(3,5)$ is isomorphic to $\Delta(2,5)$, and $\operatorname{Dr}(3,5)=\operatorname{Gr}(3,5) \cong \operatorname{Gr}(2,5)$ is isomorphic to the Petersen graph (considered as a one-dimensional polytopal complex). Also in this case the result can be verified directly. This establishes the basis of our induction, and we now assume $n \geq 6$.

Let $T$ be an arrangement of $n$ metric trees with tree metrics $\delta_{1}, \delta_{2}, \ldots, \delta_{n}$. In view of the axiom (2), the following map $\pi:[n]^{3} \rightarrow \mathbb{R} \cup\{\infty\}$ is well-defined:

$$
\pi(i, j, k)= \begin{cases}\delta_{i}(j, k)=\delta_{j}(k, i)=\delta_{k}(i, j) & \text { if } i, j, k \text { are pairwise distinct } \\ \infty & \text { otherwise }\end{cases}
$$

In order to show that $\pi$ is a tropical Plücker vector we have to verify that the minimum

$$
\min \left\{\pi_{h i j}+\pi_{h k l}, \pi_{h i k}+\pi_{h j l}, \pi_{h i l}+\pi_{h j k}\right\}
$$

is attained at least twice, for any pairwise distinct $h, i, j, k, l \in[n]$. Now, since $n \geq 6$, each 5 -tuple in $[n]$ is already contained in some deletion, and hence the desired property is satisfied by induction. We conclude that the restriction of the map $\pi$ to increasing triples $i<j<k$ is a finite tropical Plücker vector, that is, it is an element of $\operatorname{Dr}(3, n)$. By Proposition 3.1 the map $\pi$ defines a matroid subdivision $\Sigma(T)$ of $\Delta(3, n)$. 
Consider any metric tree arrangement $T^{\prime}$ that is equivalent to $T$. The maps $\pi$ and $\pi^{\prime}$ associated with $T$ and $T^{\prime}$ respectively clearly lie in the same cone of the Plücker fan structure on $\operatorname{Dr}(3, n)$. What we must prove is that they are also in the same cone of the secondary fan structure on $\operatorname{Dr}(3, n)$. Equivalently, we must show that $\Sigma\left(T^{\prime}\right)=\Sigma(T)$.

Suppose the secondary fan structure on $\operatorname{Dr}(3, n)$ is strictly finer than the Plücker fan structure. Then there is a regular matroid subdivision $\Sigma$ of $\Delta(3, n)$ whose secondary cone $S(\Sigma)$ is strictly contained in the corresponding cone $P(\Sigma)$ of tropical Plücker vectors. We fix a weight function $\lambda$ in the boundary of $S(\Sigma)$ which is contained in the interior of $P(\Sigma)$. The matroid subdivision induced by $\lambda$ is denoted by $\bar{\Sigma}$. By construction $\Sigma$ strictly refines $\bar{\Sigma}$, and by induction we can assume that $\Sigma$ and $\bar{\Sigma}$ induce the same subdivision on the entire boundary of $\Delta(3, n)$. Due to Proposition 4.3 we have that $\Sigma=\bar{\Sigma}$, and this completes our proof.

We saw in Proposition 3.3 that each regular subdivision of $\Delta_{2} \times \Delta_{n-4}$ is induced by a regular matroid subdivision of $\Delta(3, n)$. This implies that $\operatorname{Dr}(3, n)$ contains a distinguished $(2 n-9)$-dimensional sphere, dual to the secondary polytope of $\Delta_{2} \times \Delta_{n-4}$, which parameterizes all arrangements of $n-3$ lines in the tropical plane $\mathbb{T P}^{2}$. It has the following nice description in terms of tree arrangements. Let $L_{1}, L_{2}, \ldots, L_{n-3}$ be the $n-3$ lines and let $L_{x}, L_{y}, L_{z}$ denote the three boundary lines of $\mathbb{T P}^{2}$. Each of these $n$ lines translates into a tree. The tree for $L_{x}$ is obtained by branching off the leaves $\{1,2, \ldots, n-3\}$ on the path between leaves $y$ and $z$, in the order in which the $L_{j}$ intersect $L_{x}$. The trees for $L_{y}$ and $L_{z}$ are analogous. The tree for $L_{i}$ has one distinguished node with long branches to the three special leaves $x, y$ and $z$. Along the path from the distinguished node to leaf $x$ we branch off additional leaves $j$ for each line $L_{j}$ that intersects the line $L_{i}$ in its $x$-halfray. This branching takes place in the order in which the lines $L_{j}$ intersect $L_{j}$. In this manner, every arrangement of $n-3$ lines in $\mathbb{T P}^{2}$ translates into an arrangement of $n$ trees.

The same construction also applies to arrangements of $n-3$ tropical pseudolines in $\mathbb{T P}^{2}$ as defined by Ardila and Develin [2]. We shall now describe this in terms of lozenge tilings as in [26]. Let $\Sigma$ be any polytopal subdivision of $\Delta_{2} \times \Delta_{n-4}$. The Cayley Trick encodes $\Sigma$ as a mixed subdivision $M(\Sigma)$ of $(n-3) \Delta_{2}$, a regular triangle of side length $n-3$. By [26, Theorem 3.5] the mixed subdivisions of dilated triangles are characterized as those polygonal subdivisions whose cells are tiled by lozenges and upward triangles (with unit edge lengths). Here a lozenge is a parallelogram which is the union of one upward triangle and one downward triangle. We call a mixed cell even if it can be tiled by lozenges only. Those which need an upward triangle in any tiling are odd. A counting argument now reveals that each mixed subdivision of $(n-3) \Delta_{2}$ contains up to $n-3$ odd polygonal cells.

Proposition 4.5. Each polytopal subdivision $\Sigma$ of $\Delta_{2} \times \Delta_{n-4}$, or each mixed subdivision $M(\Sigma)$ of the triangle $(n-3) \Delta_{2}$, determines an abstract arrangement $T(\Sigma)$ of $n$ trees.

Proof. Assume that $\Sigma$ is a triangulation. Equivalently, $M(\Sigma)$ has exactly $n-3$ odd cells, all of which are upward triangles, and the even cells are lozenges. Placing a labeled node into each upward triangle defines a tree in the dual graph of $\Sigma$. Each of its three branches 
consists of the edges in $M(\Sigma)$ which are in the same parallel class as one fixed edge of that upward triangle. Two opposite edges in a lozenge are parallel, and the parallelism that we refer to is the transitive closure of this relation. Each parallel class of edges extends to the boundary of the triangle $(n-3) \Delta_{2}$. Doing so for all the upward triangles, we obtain an arrangement of tropical pseudo-lines [2]. Each of these is subdivided by the intersection with the other tropical pseudo-lines. We further add the three boundary lines of the big triangle to the arrangement. This specifies an abstract tree arrangement $T(\Sigma)$. Note that the trees in the arrangement partition the dual graph of the lozenge tiling.
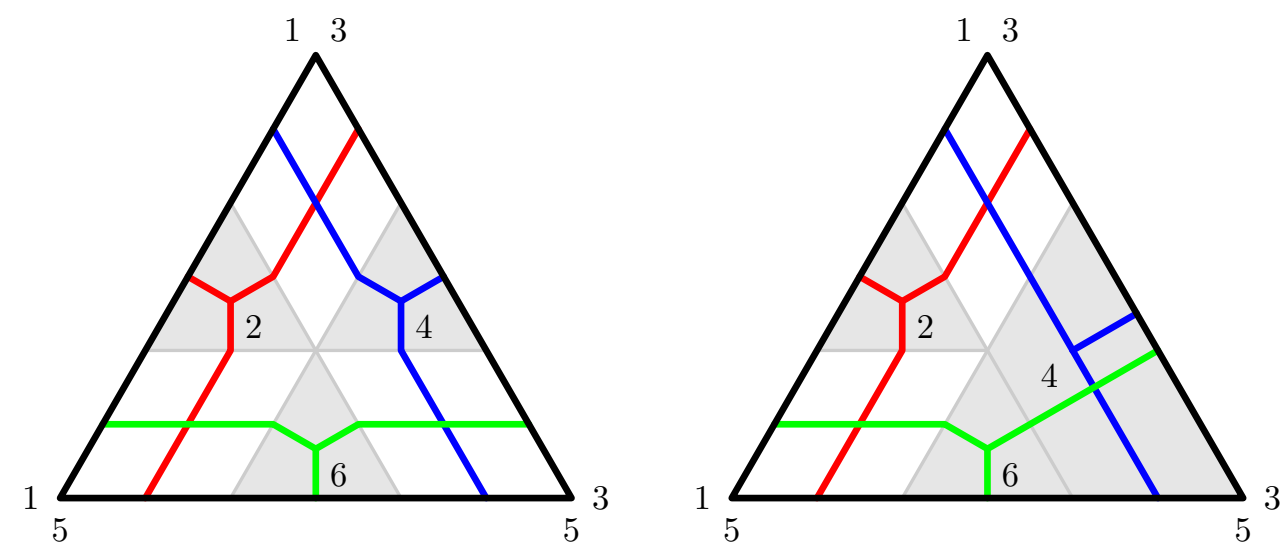

Figure 5: Mixed subdivisions of $3 \Delta_{2}$ and abstract arrangements of six trees.

Now we consider the situation where $\Sigma$ is not a triangulation, so $M(\Sigma)$ is a coarser mixed subdivision of $(n-3) \Delta_{2}$. We shall associate a tree arrangement with $M(\Sigma)$. Pick any triangulation $\Sigma^{\prime}$ which refines $\Sigma$. Then by the above procedure we have an abstract tree arrangement $T\left(\Sigma^{\prime}\right)$ induced by $\Sigma^{\prime}$. Then, as $\Sigma^{\prime}$ refines $\Sigma$, one can contract edges in the trees of the arrangement $T\left(\Sigma^{\prime}\right)$. In this way one also arrives at an abstract arrangement of $n$ trees. Three of them correspond to the boundary lines of $(n-3) \Delta_{2}$. The $n-3$ non-boundary trees are assigned to the $\leq n-3$ odd cells. Each cell is assigned at least one tree. We note that $T(\Sigma)$ might depend on the choice of the triangulation $\Sigma^{\prime}$.

Example 4.6. Let $n=6$ and consider the two mixed subdivisions of $3 \Delta_{2}$ shown in Figure 5. The left one is a lozenge tiling which encodes a regular triangulation of $\Delta_{2} \times \Delta_{2}$, here regarded as the vertex figure of $\Delta(3,6)$ at $e_{135}$. There are precisely three upward triangles, and each of them corresponds to a tree. Moreover, the three sides of the big triangle encode three more trees. Using the notation of Figure 6, this abstract tree arrangement equals

$$
34256,34156,12456,12356,12634,12534 \text {. }
$$

The tiling of $3 \Delta_{2}$ on the right in Figure 5 is a mixed subdivision which coarsens the lozenge tiling discussed above. It corresponds to the abstract tree arrangement

$$
34256,34156,12(456), 12(356), 12634,12534 \text {. }
$$

The tree $a b(c d e)$ is obtained from the tree $a b c d e$ by contracting the interior edge between $c$ and the pair de. The odd polygonal cells (shaded in Figure 5) correspond to trees. 


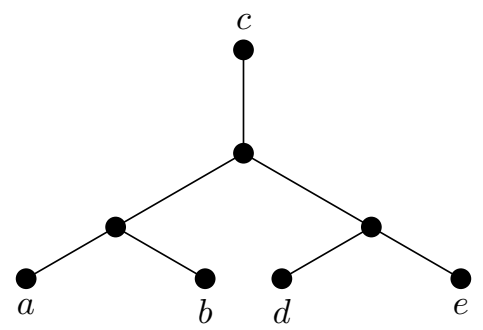

Figure 6: We use the notation abcde for this tree on five labeled leaves.

Example 4.7. An example of a non-regular matroid subdivision arises from the lozenge tiling of $6 \Delta_{2}$ borrowed from [26] and shown in Figure 3. This picture translates into the abstract arrangement of nine trees in Table 1 . The corresponding matroid subdivision of $\Delta(3,9)$ is not regular. Equivalently, the Dressian $\operatorname{Dr}(3,9)$ has no cell for this tree arrangement.

Table 1: Abstract arrangement of nine caterpillar trees on eight leaves encoding a matroid subdivision of $\Delta(3,9)$ which is not regular; see Figure 3 . The notation for caterpillar trees is explained in Figure 8 below.

\begin{tabular}{lll} 
Tree 1: $\mathrm{C}(24,6598,37)$ & Tree 2: $\mathrm{C}(14,5768,39)$ & Tree 3: $\mathrm{C}(17,5846,29)$ \\
Tree 4: $\mathrm{C}(12,6579,38)$ & Tree 5: $\mathrm{C}(26,4198,37)$ & Tree 6: $\mathrm{C}(14,5729,38)$ \\
Tree 7: $\mathrm{C}(13,5894,26)$ & Tree 8: $\mathrm{C}(15,7346,29)$ & Tree 9: $\mathrm{C}(15,7468,23)$ \\
\hline
\end{tabular}

Remark 4.8. There are 187 lozenge tilings of $4 \Delta_{2}$, each representing 24 triangulations of $\Delta_{3} \times \Delta_{2}$ via the 4 ! = 24 ways of labeling the upward triangles. Each lozenge tiling defines an arrangement of seven trees that indexes a maximal cell of $\operatorname{Dr}(3,7)$. In other words, the polytopal 5-sphere dual to the secondary polytope of $\Delta_{2} \times \Delta_{3}$ has $4488=187 \cdot 24$ facets, and embeds as a subcomplex into $\operatorname{Dr}(3,7)$. It is instructive to study this subcomplex by browsing our website for $\operatorname{Dr}(3,7)$. For example, the tropical plane of type 89 on our website corresponds to Figure 4 in [2].

Remark 4.9. Another important sphere sitting inside the Grassmannian $\operatorname{Gr}(d, n)$, and hence in the Dressian $\operatorname{Dr}(d, n)$, is the positive Grassmannian $\operatorname{Gr}^{+}(d, n)$, due to Speyer and Williams [30]. A natural next step would be to introduce and study the positive Dressian $\operatorname{Dr}^{+}(d, n)$. Generalizing [30, $\left.\S 5\right]$, the positive Dressian $\operatorname{Dr}^{+}(3, n)$ would parameterize metric arrangements of planar trees. This space contains the $(2 n-9)$-dimensional sphere $\operatorname{Gr}^{+}(3, n)$. It would be interesting to know whether this inclusion is a homotopy equivalence, to explore relations with cluster algebras, and to extend the computation of $\mathrm{Gr}^{+}(3,7)$ presented in [30]. Incidentally, there is a misprint in the right part of [30, Table 2]: the eleventh inequality should be " $-x_{5} \leq-14$ " instead of " -17 ". With this correction, we independently verified the $f$-vector and the rays of its normal fan $F_{3,7}$. 


\section{Tropical Planes}

We are now finally prepared to answer the question raised in the title of this paper. Tropical planes are contractible polyhedral surfaces that are dual to the regular matroid subdivisions of $\Delta(3, n)$. Consider any vector $p$ in $\mathbb{R}^{\left(\begin{array}{l}n \\ 3\end{array}\right)}$ that lies in the Dressian $\operatorname{Dr}(3, n)$. The associated tropical plane $L_{p}$ in $\mathbb{T P}^{n-1}$ is the intersection of the tropical hyperplanes

$$
\mathbb{T}\left(p_{i j k} x_{l}+p_{i j l} x_{k}+p_{i k l} x_{j}+p_{j k l} x_{i}\right)
$$

as $\{i, j, k, l\}$ ranges over all 4 -element subsets of $[n]$. By a tropical plane we mean any subset of $\mathbb{T P}^{n-1}$ which has the form $L_{p}$ for some $p \in \operatorname{Dr}(3, n)$. The tropical plane $L_{p}$ is realizable as the tropicalization of a classical plane in $\mathbb{P}_{\mathbb{K}}^{n-1}$ if and only if $p \in \operatorname{Gr}(3, n)$. The plane $L_{p}$ is called series-parallel if each cell in the corresponding matroid subdivision of $\Delta(3, n)$ is the graphic matroid of a series-parallel graph. Results of Speyer [27, 28] imply:

Proposition 5.1. Let $L$ be a tropical plane in $\mathbb{T P}^{n-1}$ with $f_{0}(L)$ vertices, $f_{1}^{b}(L)$ bounded edges, $f_{1}^{u}(L)$ unbounded edges, $f_{2}^{b}(L)$ bounded 2 -cells and $f_{2}^{u}(L)$ unbounded 2 -cells. Then

$$
\begin{aligned}
f_{0}(L) \leq(n-2)(n-3) / 2, & f_{1}^{b}(L) \leq(n-4)(n-3), \quad f_{1}^{u}(L) \leq n(n-3), \\
& f_{2}^{b}(L) \leq(n-4)(n-5) / 2, \quad f_{2}^{u}(L) \leq 3 n(n-1) / 2 .
\end{aligned}
$$

These five inequalities are equalities if and only if $L$ is a series-parallel plane.

The unbounded edges and 2-cells of a tropical plane correspond to the nodes and edges of the $n$ trees in the corresponding tree arrangement. Suppose the trees are trivalent. Then each tree has $n-1$ leaves and $n-3$ nodes, for a total of $f_{1}^{u}(L)=n(n-3)$ nodes. Moreover, each tree has $n-4$ interior edges and $n-1$ pendent edges. The latter are double-counted. This explains the number $f_{2}^{u}(L)=n(n-4)+n(n-1) / 2$ of edges in the tree arrangement representing $L$. To understand this situation geometrically, we identify $\mathbb{T P}^{n-1}$ with an $(n-1)$-simplex, and we note that the tree arrangement is obtained geometrically as the intersection $L \cap \partial \mathbb{T} \mathbb{P}^{n-1}$ of $L$ with the boundary of that simplex.

The first answer to our question of how to draw a tropical plane is given by Theorem 4.4: simply draw the corresponding tree arrangement. This answer has the following interpretation as an algorithm for enumerating all tropical planes. To draw all (generic) planes $L$ in $\mathbb{T} \mathbb{P}^{n-1}$, we first list all trees on $n-1$ labeled leaves. Each labeled tree occurs in $n$ relabelings corresponding to the sets $[n] \backslash\{1\},[n] \backslash\{2\}, \ldots,[n] \backslash\{n\}$ of labels. Inductively, one enumerates all arrangements of $4,5, \ldots, n$ trees. This naive approach works well for $n \leq 6$. The result of the enumeration is that, up to relabeling and restricting to trivalent trees, there are precisely seven abstract tree arrangements for $n=6$. They are listed in Table 2. Each tree is written as abcde, the notation introduced in Figure 6. We then check that each of the seven abstract tree arrangements supports a metric tree arrangement, and we conclude that $\operatorname{Dr}(3,6)$ has seven maximal cells modulo the natural action of the group $\mathrm{S}_{6}$. The names for the seven types of generic planes are the same as in $[29, \S 5]$ and in Figure 1.

It is easy to translate the seven rows in Table 2 into seven pictures of tree arrangements. For example, the representative for type FFFGG in the last row coincides with (3) and 
Table 2: The trees corresponding to the seven types of tropical planes in $\mathbb{T P}^{5}$.

\begin{tabular}{lccccccr}
\hline Type & Tree 1 & Tree 2 & Tree 3 & Tree 4 & Tree 5 & Tree 6 & Orbit Size \\
\hline EEEE & 23645 & 13546 & 12456 & 15326 & 14236 & 24135 & 30 \\
EEEG & 26534 & 16534 & 14256 & 13256 & 12346 & 12345 & 240 \\
EEFF(a) & 25634 & 15634 & 12546 & 12536 & 12634 & 12534 & 90 \\
EEFF(b) & 25634 & 15634 & 12645 & 12635 & 12634 & 12534 & 90 \\
EEFG & 25634 & 15634 & 24156 & 23156 & 12634 & 12534 & 360 \\
EFFG & 34256 & 34156 & 12645 & 12635 & 12634 & 12534 & 180 \\
FFFGG & 34256 & 34156 & 12456 & 12356 & 12634 & 12534 & 15 \\
\hline
\end{tabular}

its picture appears on the left side in Figure 5. It can be checked in the pictures that each of the seven tree arrangements has $f_{1}^{u}(L)=18$ nodes and $f_{2}^{u}(L)=27$ edges.

The second answer to our question of how to draw a tropical plane is given by Figure 1: simply draw and label the bounded cells. The planes $L$ in the last six rows of Table 2 are series-parallel. Here, the complex of bounded cells in $L$ has $f_{0}(L)=6$ nodes, $f_{1}^{b}(L)=6$ edges and $f_{2}^{b}(L)=1$ two-dimensional cell. The first type EEEE is not series-parallel: its bounded complex is one-dimensional, with four edges and five nodes.

Each node of (the complex of bounded cells of) a tropical plane $L$ is labeled by a connected rank-3-matroid. This is the matroid whose matroid polytope is dual to that node in the matroid subdivision of $\Delta(3, n)$ given by $L$. For $n=6$ only three classes of matroids occur as node labels of generic planes. These matroids are denoted $\{A, B, C, D\}$, $[A, B, C, D](E)$, or $\langle A ; a ;(b, c, d, e)\rangle$. Here capital letters are non-empty subsets of and lower-case letters are elements of the set $\{1,2,3,4,5,6\}$. All three matroids are graphical. The corresponding graphs are shown in Figure 7. Note that an edge labeled with a set of $l$ points should be considered as $l$ parallel edges each labeled with one element of the set.
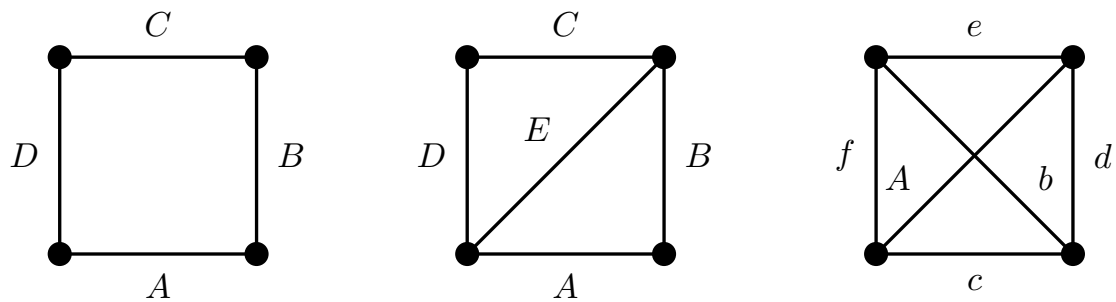

Figure 7: The graphic matroids corresponding to the labels $\{A, B, C, D\},[A, B ; C, D](E)$ and $\langle A ; b ;(c, d, e, f)\rangle$ used for the nodes in Figure 1.

The underlying graph of the matroid $\langle A ; b ;(c, d, e, f)\rangle$ is the complete graph $K_{4}$. The set $A$ is a singleton, and thus its automorphism group is the full symmetric group $\mathrm{S}_{4}$ of order 24 acting on the four nodes of $K_{4}$. This matroid occurs in the unique orbit of planes (of type EEEE) in $\mathbb{T} \mathbb{P}^{6}$ whose bounded parts are not two-dimensional. The series-parallel planes use only the matroids $\{A, B, C, D\}$ and $[A, B ; C, D](E)$ for their labels.

The third answer to our question is the synthesis of the previous two: draw both the bounded complex and the tree arrangement. The two pictures can be connected, 
by linking each node of $L$ to the adjacent unbounded rays and 2-cells. This leads to an accurate diagram of the tropical plane $L$. The reader might enjoy drawing these connections between the seven rows of Table 2 and the seven pictures in Figure 1.

The analogous complete description for $n=7$ is a main contribution of this paper. Based on the computational results in Section 2, we prepared an online census of $\operatorname{Gr}(3,7)$ and $\operatorname{Dr}(3,7)$, with a picture for each bounded complex. This is posted at our website

Www . uni-math.gwdg.de/jensen/Research/G3_7/grassmann3_7.html.
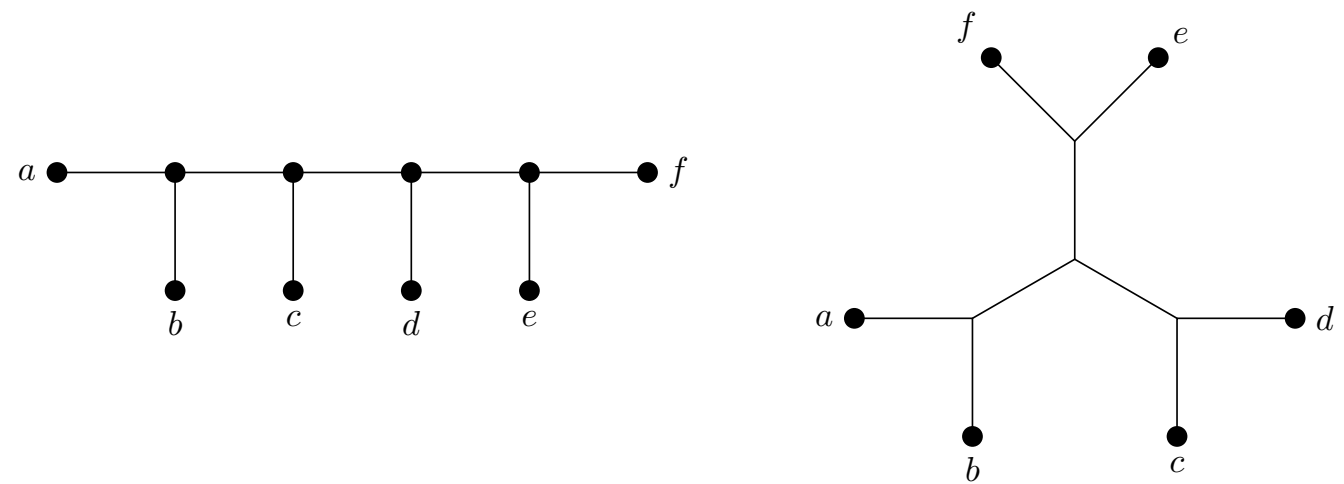

Figure 8: Caterpillar tree $\mathrm{C}(a b, c d, e f)$ and snowflake tree $\mathrm{S}(a b, c d, e f)$.

The maximal cells of the Dressian $\operatorname{Dr}(3,7)$ correspond to arrangements of seven trivalent trees. As part of our computations, we found that for $n=7$ there is no difference between abstract tree arrangements and metric tree arrangements: nothing like Example 4.7 exists in this case. To draw the tree arrangements, we note that there are two distinct trivalent trees on six leaves. These are the caterpillar and the snowflake trees depicted in Figure 8. Caterpillar trees exist for all $n \geq 5$, and are encoded using a natural generalization of the notation in Figure 6. Note, for instance, the caterpillars with eight leaves in Table 1.

We conclude with a brief discussion of the 94 generic planes depicted on our website. Four types of node labels occur in the Dressian $\operatorname{Dr}(3,7)$. First of all, the matroids $\{A, B, C, D\},[A, B, C, D](E)$, and $\langle A ; b ;(c, d, e, f)\rangle$ appear again. Here capital letters are non-empty subsets of and lower-case letters are elements of $\{1,2, \ldots, 7\}$. The other matroid which occurs is the Fano matroid $\mathcal{F}_{3}$ arising from the projective plane $\mathrm{PG}_{2}(2)$; see Figure 2 (left). It corresponds to the six-dimensional cells of $\operatorname{Dr}(3,7)$ generated by seven vertex splits. Each such 6-cell admits seven coarsenings arising from omitting one of the seven splits. These coarsenings correspond to the non-Fano matroid; see Figure 2 (right).

\section{$6 \quad$ Restricting to Pappus}

The Grassmannian $\operatorname{Gr}(d, n)$ is a tropical variety and the Dressian $\operatorname{Dr}(d, n)$ is a tropical prevariety. We now consider these two fans inside the tropical projective space $\mathbb{T P}\left(\begin{array}{l}n \\ d\end{array}\right)-1$. 
That projective space is a simplex, and it makes sense to study their intersections with each (relatively open) face of $\mathbb{T P}\left(\begin{array}{l}n \\ d\end{array}\right)-1$. That intersection is non-empty only if the face corresponds to a matroid $\mathcal{M}$ of rank $d$ on $[n]$. This leads to the following relative versions of our earlier definitions.

We define the Grassmannian $\operatorname{Gr}(\mathcal{M})$ of a matroid $\mathcal{M}$ to be the tropical variety defined by the ideal $I_{\mathcal{M}}$ which is obtained from the Plücker ideal by setting to zero all variables $p_{X}$ where $X$ is not a basis of $\mathcal{M}$. We define the Dressian $\operatorname{Dr}(\mathcal{M})$ to be the tropical prevariety given by the set of quadrics which are obtained from the quadratic Plücker relations by setting to zero all variables $p_{X}$ where $X$ is not a basis of $\mathcal{M}$. Equivalently, in the language of $[8,9]$, the Dressian $\operatorname{Dr}(\mathcal{M})$ is the set of all real-valued valuations of the matroid $\mathcal{M}$. As before, $\operatorname{Gr}(\mathcal{M})$ is a subfan of the Gröbner fan of $I_{\mathcal{M}}$, the Dressian $\operatorname{Dr}(\mathcal{M})$ is a subfan of the secondary fan of the matroid polytope of $\mathcal{M}$, and we regard these fans as polyhedral complexes after removing the lineality space and intersecting with a sphere. Note that the cells of $\operatorname{Dr}(\mathcal{M})$ are in bijection with the regular matroid subdivisions of the matroid polytope of $\mathcal{M}$. The Grassmannian $\operatorname{Gr}(d, n)$ and the Dressian $\operatorname{Dr}(d, n)$ discussed in the previous sections are special cases where $\mathcal{M}$ is the uniform matroid of rank $d$ on $n$ elements. The Dressian $\operatorname{Dr}(d, n)$ contains the Dressians of all matroids of rank $d$ on $n$ elements as subcomplexes at infinity.

In this final section we examine these concepts in detail for one important example, namely, we take $\mathcal{M}$ to be the Pappus matroid. Here $d=3, n=9, \mathcal{M}$ has 75 bases, and the non-bases are the nine lines in the Pappus configuration shown in Figure 9:

$$
\text { 123, 148, 159, 247, 269, 357, 368, 456, } 789 \text {. }
$$

The ideal $I_{\mathcal{M}}$ is the ideal in the polynomial ring in 75 variables obtained from the Plücker ideal by setting the corresponding nine Plücker coordinates to zero: $p_{123}=\cdots=p_{789}=0$.
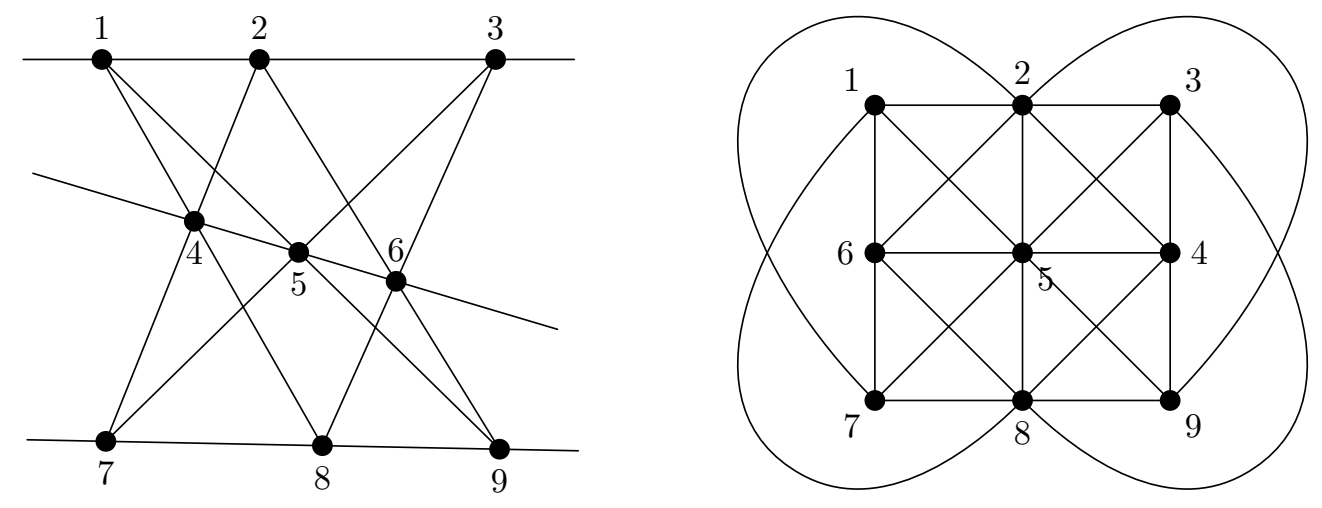

Figure 9: Pappus configuration (left) and Hessian configuration (right).

The realization space of the Pappus configuration modulo projective transformations is two-dimensional, and the Grassmannian $\operatorname{Gr}(\mathcal{M})$ is the corresponding tropical surface. We shall determine the underlying graph and how it embeds into the Dressian $\operatorname{Dr}(\mathcal{M})$.

Proposition 6.1. The Grassmannian $\operatorname{Gr}(\mathcal{M})$ of the Pappus matroid $\mathcal{M}$ is a graph with 19 
nodes and 30 edges. One of the nodes gets replaced by a triangle in the Dressian $\operatorname{Dr}(\mathcal{M})$. The Dressian $\operatorname{Dr}(\mathcal{M})$ is a simplicial complex with 18 vertices, 30 edges and one triangle.

Proof. What follows is a detailed description, first of $\operatorname{Gr}(\mathcal{M})$ and later of $\operatorname{Dr}(\mathcal{M})$. The Grassmannian $\operatorname{Gr}(\mathcal{N})$ has three split nodes, represented by the bases 167, 258 and 349 of the Pappus matroid $\mathcal{M}$. These three bases are characterized by the property that their two-element subsets form two-point lines. The corresponding matroid subdivisions are vertex splits, and they are the only splits of the matroid polytope $P_{\mathcal{N} \text {. }}$. The three split vertices have valence four, and they are connected to a special trivalent core node $C$.

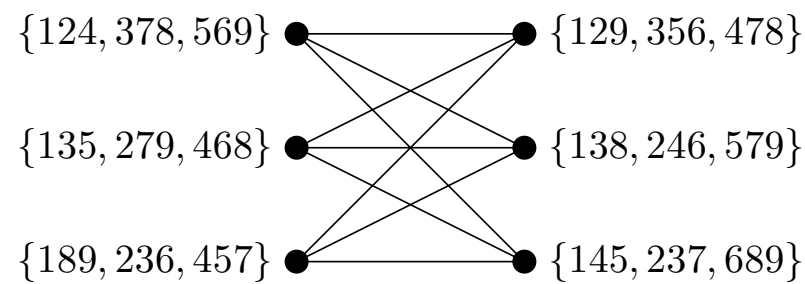

Figure 10: Complete bipartite graph formed from the Graves triads.

The remaining 15 nodes are all trivalent in $\operatorname{Gr}(\mathcal{M})$, and their subgraph corresponds to the vertices and edges of the complete bipartite graph $K_{3,3}$. The six vertices of $K_{3,3}$ correspond to six Graves nodes, one for each of the Graves triads in the Pappus configuration. A Graves triad is a partition of the nine points into three bases whose two-element subsets span three-point lines. Each Graves node defines a matroid subdivision with three maximal cells. The three corresponding matroids have 52 bases, and they are obtained geometrically by merging together the three points in a triple of the Graves triad. For example, the first matroid in the subdivision defined by the Graves triad $\{145,237,689\}$ is obtained from the Pappus matroid by making 1, 4 and 5 parallel elements.

The six Graves triads form the vertices of the graph $K_{3,3}$ shown in Figure 10 . On each of the nine edges lies a connector node of $\operatorname{Gr}(\mathcal{M})$, which is between two Graves nodes and also adjacent to one of the three split nodes. Each connector node defines a matroid subdivision with seven maximal cells. The number of bases of these seven matroids are 36, 36, 36, 40, 40, 40, 51. For a concrete example consider the two adjacent Graves triads $\{145,237,689\}$ and $\{189,236,457\}$. On the edge between them in $K_{3,3}$ we find a connector node which is also adjacent to the split node 167 . The seven matroids in the matroid subdivision of $P_{\mathcal{M}}$ represented by that connector node are the rows in the following table:

We now come to the Dressian $\operatorname{Dr}(\mathcal{M})$ of the Pappus matroid $\mathcal{M}$. This is a nonpure complex whose facets are one triangle and 27 edges. It is obtained from $\operatorname{Gr}(\mathcal{M})$ by removing the core node and replacing it with the core triangle whose nodes are the split nodes 167, 258 and 349. Thus $\operatorname{Dr}(\mathcal{M})$ has 18 vertices, 30 edges and one triangle. The core triangle of $\operatorname{Dr}(\mathcal{M})$ represents the matroid subdivision which is obtained from the Pappus matroid polytope by slicing off the three vertices 167, 258 and 349. What remains is the matroid polytope of the Hessian configuration shown in Figure 9. This is the matroid associated with the affine plane over the field $\mathbb{G F}(3)$ with three elements. Collinearity of any eleven of its twelve triples implies collinearity of the last. It is this incidence theorem 


\begin{tabular}{cc}
\hline number of bases & parallelism classes \\
\hline 51 & $\{8,9\},\{2,3\},\{4,5\}$ \\
40 & $\{2,3,6,7\}$ \\
40 & $\{1,4,5,7\}$ \\
40 & $\{1,6,8,9\}$ \\
36 & $\{4,5,7\},\{6,8,9\}$ \\
36 & $\{1,4,5\},\{2,3,6\}$ \\
36 & $\{1,8,9\},\{2,3,7\}$ \\
\hline
\end{tabular}

which explains the difference between $\operatorname{Gr}(\mathcal{M})$ and $\operatorname{Dr}(\mathcal{M})$. An algebraic witness is offered by the expression

$$
p_{289} p_{389} p_{489} p_{569} p_{589} \underline{p_{167}}-p_{189} p_{389} p_{489} p_{569} p_{679} \underline{p_{258}}+p_{189} p_{289} p_{569} p_{589} p_{678} \underline{p_{349}} .
$$

This trinomial lies in the Pappus ideal $I_{\mathcal{M}}$, and it shows that the tropical variety of $I_{\mathcal{M}}$ does not contain the entire triangular cone spanned by the basis vectors $e_{167}, e_{258}, e_{349}$. As the minimum must be attained at least twice, we conclude that, locally on the core triangle of the Dressian $\operatorname{Dr}(\mathcal{N})$, the Grassmannian $\operatorname{Gr}(\mathcal{M})$ looks like a tropical line.

\section{References}

[1] V. Alexeev: Weighted Grassmannians and stable hyperplane arrangements, 2008, arXiv:0806.0881.

[2] F. Ardila and M. Develin: Tropical hyperplane arrangements and oriented matroids, to appear in Mathematische Zeitschrift, arXiv:0706.2920.

[3] H.-J. Bandelt and A. Dress: A canonical decomposition theory for metrics on a finite set, Adv. Math. 92 (1992), no. 1, 47-105.

[4] R. Bieri and J. R. J. Groves: The geometry of the set of characters induced by valuations, J. Reine Angew. Math. 347 (1984), 168-195.

[5] A. Björner: Topological methods, Handbook of Combinatorics, Vol. 1, 2, 1819-1872, Elsevier, Amsterdam, 1995.

[6] T. Bogart, A. Jensen, D. Speyer, B. Sturmfels, and R. Thomas: Computing tropical varieties, J. Symbolic Comput. 42 (2007) 54-73.

[7] J. De Loera, J. Rambau, and F. Santos: Triangulations: Structures and Algorithms, Algorithms and Computations in Mathematics Springer Verlag, Heidelberg, 2009.

[8] A. Dress and W. Wenzel: Perfect matroids, Adv. Math. 91 (1992), no. 2, 158-208.

[9] _ : Valuated matroids, Adv. Math. 93 (1992), no. 2, 214-250.

[10] J.-G. Dumas, F. Heckenbach, B. D. Saunders, and V. Welker: Simplicial Homology, a (proposed) GAP share package, http://www.cis.udel.edu/ dumas/Homology/. 
[11] E. M. Feichtner and B. Sturmfels: Matroid polytopes, nested sets and Bergman fans, Portugaliae Math. 62 (2005), no. 4, 437-468.

[12] K. Fukuda: Cddlib Reference Manual, cddlib version 94, (2005), http://www.ifor.math.ethz.ch/ fukuda/cdd_home/cdd.html

[13] E. Gawrilow and M. Joswig: polymake: a framework for analyzing convex polytopes, Polytopes-combinatorics and computation (Oberwolfach, 1997), DMV Sem., vol. 29, Birkhäuser, Basel, 2000, pp. 43-73.

[14] I. M. Gel'fand, M. Goresky, R. D. MacPherson, and V. V. Serganova: Combinatorial geometries, convex polyhedra, and Schubert cells, Adv. Math. 63 (1987), no. 3, 301316.

[15] P. Hacking: The homology of tropical varieties, Collectanea Mathematica 59 (2008), $263-273$.

[16] P. Hacking, S. Keel, and J. Tevelev: Compactification of the moduli space of hyperplane arrangements, J. Algebraic Geom. 15 (2006) 657-680.

[17] S. Herrmann and M. Joswig: Splitting polytopes, Münster J. Math., 1 (2008), 109 142.

[18] A. Jensen: Gfan-a software package for Gröbner fans and tropical varieties, www. math.tu-berlin.de/ jensen/software/gfan/gfan.html.

[19] D. R. Grayson and M. E. Stillman: Macaulay 2, a software system for research in algebraic geometry, http://www .math.uiuc.edu/Macaulay2/.

[20] M. M. Kapranov: Chow quotients of Grassmannians. I, I. M. Gel'fand Seminar, 29-110, Adv. Soviet Math., 16, Part 2, Amer. Math. Soc., Providence, RI, 1993.

[21] S. Keel and J. Tevelev: Geometry of Chow quotients of Grassmannians, Duke Math. J. 134 (2006), 259-311.

[22] H. Markwig and J. Yu: The space of tropically collinear points is shellable, Collect. Math. 60 (2009), no. 1, 63-77.

[23] G. Mikhalkin: Introduction to Tropical Geometry, IMPA notes and book manuscript, arXiv:0709.1049.

[24] L. Pachter and B. Sturmfels: Algebraic Statistics for Computational Biology, Cambridge University Press, 2005.

[25] J. Richter-Gebert, B. Sturmfels, and T. Theobald: First steps in tropical geometry, Idempotent mathematics and mathematical physics, 289-317, Contemp. Math., 377, Amer. Math. Soc., Providence, RI, 2005.

[26] F. Santos: The Cayley trick and triangulations of products of simplices, Integer points in polyhedra - geometry, number theory, algebra, optimization, 151-177, Contemp. Math., 374, Amer. Math. Soc., Providence, RI, 2005.

[27] D. Speyer: Tropical linear spaces, SIAM J. Discrete Math. 22 (2008), no. 4, 15271558. 
[28] _ _ A matroid invariant via K-theory of the Grassmannian, to appear in Adv.in Math., arXiv:math.AG/0603551.

[29] D. Speyer and B. Sturmfels: The tropical Grassmannian, Adv. Geometry 4 (2004), 389-411.

[30] D. Speyer and L. Williams: The tropical totally positive Grassmannian, J. Algebraic Combin. 22 (2005), 189-210.

[31] N. White (ed.), Theory of Matroids, Encyclopedia of Mathematics and its Applications, vol. 26, Cambridge University Press, Cambridge, 1986.

[32] R. Wunderling: Paralleler und objektorientierter Simplex-Algorithmus, PhD thesis, Technische Universität Berlin, 1996, available at http://www.zib.de/ Publications/abstracts/TR-96-09/. 